\title{
Geomorphologische, palynologische und archäologische Bei- träge zur holozänen Landschaftsgeschichte im Müritzgebiet (Mecklenburg-Vorpommern)
}

\author{
Knut Kaiser, Thomas Schoknecht, Wolfgang Janke, Klaus Kloss \& Burkhard Prehn*)
}

\begin{abstract}
Kaiser, K., Schoknecht, Th., Janke, W., Kloss, K. \& PREFN, B. (2002): Geomorphologische, palynologische und archäologische Beiträge zur holozänen Landschaftsgeschichte im Müritzgebiet (Mecklenburg-Vorpommern). - Eiszeitalter und Gegenwart, 5 1: 15-32; Hannover 2002.
\end{abstract}

Keywords: Holocene, lake-level fluctuations, vegetation history, human impact

Kurzfassung: Vorgestellt werden Untersuchungen zur holozänen Landschaftsentwicklung im Gebiet der Müritz, des größten Sees der Norddeutschen Tiefebene. Geomorphologische Untersuchungen zeigen für das Holozän bis zum Mittelalter eine im Wesentlichen aufwärts gerichtete Tendenz des Seespiegels (9500 BP: $57 \mathrm{~m} \mathrm{NN}, 5400$ BP: $61 \mathrm{~m} \mathrm{NN}$, AD 1100: $61 \mathrm{~m} \mathrm{NN}$, AD 1280: 62-63 m NN, nach AD 1300: $65 \mathrm{~m} \mathrm{NN}, \mathrm{AD}$ 1788: 63,5 m NN, heute: $62 \mathrm{~m} \mathrm{NN).} \mathrm{Östlich} \mathrm{des} \mathrm{Sees}$ wurden unter Wald große Flächen mit jungen anthropogenen Erosionsspuren nachgewiesen. Vier Pollendiagramme geben einen Überblick zur Vegetationsentwicklung auf verschiedenen Standorten und bei unterschiedlich starkem menschlichen Einfluß. Die bislang im Gebiet nachgewiesenen 401 archäologischen Fundplätze datieren überwiegend in das Neolithikum, die Bronzezeit und die Slawenzeit. Die Auswirkungen des Menschen auf den See und sein Umland sowie die Qualität der Befunde stehen im Mittelpunkt der Diskussion.

\section{[Geomorphological, palynological and archaeological investigations on the Holocene landscape development in the Lake Mueritz area (Mecklenburg-Vorpommern, NEGermany)]}

\begin{abstract}
Investigations on the Holocene landscape development in the Lake Mueritz area, a landscape characterized by the largest lake $\left(117 \mathrm{~km}^{2}\right)$ in the North German Plain, are presented. A general rising tendency of the Holocene lake-level up to the Medieval time is proved by geomorphological studies (9500 BP: $57 \mathrm{~m}$ a.s.l., 5400 BP: $61 \mathrm{~m}$ a.s.l., AD 1100: $61 \mathrm{~m}$ a.s.l., AD 1280: 62$63 \mathrm{~m}$ a.s.l., after $\mathrm{AD}$ 1300: $65 \mathrm{~m}$ a.s.l., AD 1788: 63,5 m
\end{abstract}

\footnotetext{
*)Anschrift der Verfasser: Dr. Knut KaISER, Prof. Dr. Wolfgang JANKE, Universität Greifswald, Geographisches Institut, Friedrich-Ludwig-Jahn-Straße 16, D-17487 Greifswald; Dr. Thomas SaHOKNECH, Steinweg 19a, D-14532 Kleinmachnow; Dipl.-Prähist. Burkhard PRFHN, Mühlenstraße 9b, D-17039 Wulkenzin; Dr. Klaus KLoss, Seestraße 5a, D-14542 Kemnitz.
}

a.s.l., today: $62 \mathrm{~m}$ a.s.1.). Marked traces of medieval soil erosion in large wooded areas were found east of the lake shore. An overview of the Holocene vegetation history is given by means of 4 pollen diagrams. They reflect in each case a development depending on natural setting (soil, moisture, site sedimentation) and human impact. At present 401 archaeological sites were detected by mapping. They belong mainly to the Neolithic, the Bronce Age and the Slavonic period. The discussion is focused both on the human impact on the lake and its surrounding area and the quality of the records.

\section{Einführung}

Ein komplexer landschaftsgeschichtlicher Ansatz im Sinne einer lokalen Synthese von Landschaftsund Siedlungsentwicklung wurde in Norddeutschland erstmals in den 30er Jahren versucht (RusT 1937). Verschiedene Projekte unter der Federführung der Archäologie und der Palynologie zielten dann in den 70er und 80er Jahren in Niedersachsen, Schleswig-Holstein und auf Rügen in Richtung einer interdisziplinären Erforschung der kleinräumigen Landschaftsentwicklung (z.B. KossaCK et al. 1984; LANGE et al. 1986; MüUer-WIIIE et al. 1988). Schließlich verstärkten sich in der zweiten Hälfte der 80er Jahre in ganz Mitteleuropa Bestrebungen für eine interdisziplinäre Untersuchung der großräumigen Landschaftsgeschichte. Hier seien beispielsweise die mit siedlungsgeschichtlichem Ansatz initiierten Projekte am Bodensee (SaHLATERIE 1990), in SüdSchweden (BFRGIUND 1991), in West-Polen (NEWIAROWSKI et al. 1995) und an der unteren Oder (GRNGMUTH DALMER 1997) genannt. Vor dem Hintergrund aktueller Fragen zur Klimaentwicklung im Quartär wurden in den 90er Jahren verschiedene Großprojekte aufgelegt (FRENHL 1996, ANDRES 1998), wobei jeweils auch interdisziplinäre landschaftsgeschichtliche Teilvorhaben realisiert wurden. Beispielsweise konnte im Rahmen des DFG-Schwerpunktprogrammes „Wandel der Geo-Biosphäre während der letzten 15.000 Jahre" ein regionales Projekt zur spätglazialen bis frühholozänen Landschafts- und Besiedlungsgeschichte in Vorpommern durchgeführt werden (B⿺𠃊wIZ et al. 2000). 

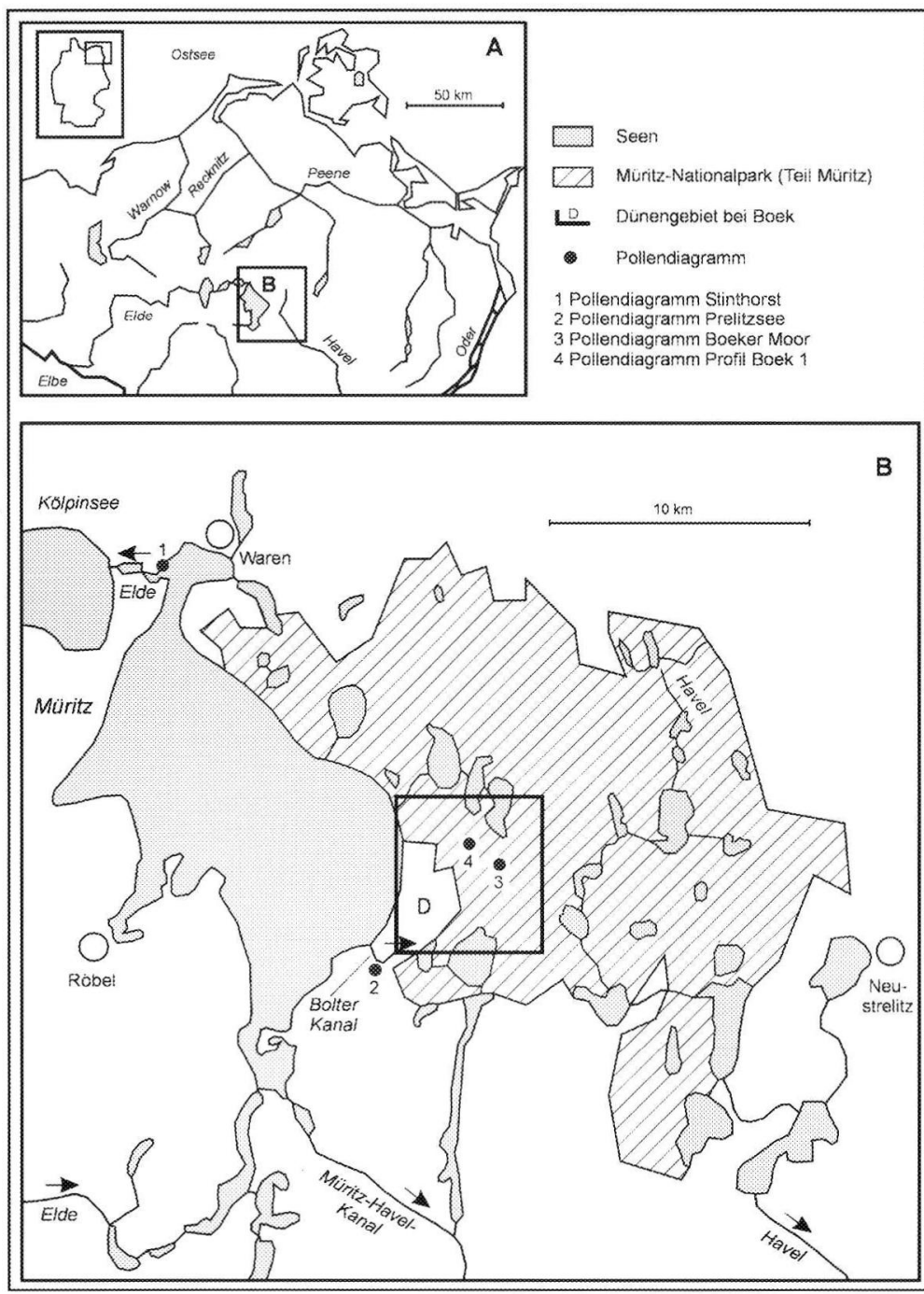

Abb. 1: Lage des Untersuchungsraumes (A) und Karte des Müritzgebietes mit Untersuchungspunkten (B).

Fig. 1: Location of the study region (A) and map of Lake Mueritz area with study sites (B).

In Mecklenburg-Vorpommern stand, bei einer bislang insgesamt unzureichenden Forschungslage, neben der Ostseeküste häufiger die Mecklenburger Seenplatte und hier vor allem das Müritzgebiet im Mittelpunkt des landschaftsgeschichtlichen Interesses (Abb. 1). Der mit ca. $117 \mathrm{~km}^{2}$ größte See des norddeutschen Tieflandes ist seit dem Ende des 18. Jh. Objekt landschaftshistorischer Erörterungen (SCHUMACHER 1790). Der Beginn einer komplexen geowissenschaftlichen Untersuchung des Sees datiert in das beginnende 20. Jh. (GEINITZ 1913); den aktuellen Wissensstand repräsentieren Arbeiten der Autoren aus den 80 er und 90 er Jahren.

Die hier vorgelegten Untersuchungen von der Müritz stammen nicht aus einem übergreifenden Projekt, sondern sind das Ergebnis von Einzelaktivitäten der Autoren. Die 1990 erfolgte Ausweisung des Müritz-Nationalparkes und der damit entstandene Bedarf an fundierten wissenschaftlichen Informationen für Parkmanagement und Umweltpädagogik führten zu einer Belebung entsprechender Forschungsaktivitäten. Da nur ein geringer Teil des neuen Materials zur Landschaftsgeschichte bislang publiziert wurde und eine notwendige Zusammenschau der Ergebnisse aussteht, soll hier der Versuch einer regionalen Synthese unternommen werden.

\section{Geomorphologische Untersuchungen}

\section{Seebildung}

Im Mittelpunkt vieler geowissenschaftlicher Arbeiten zur Müritz stand die Suche nach und die Interpretation von Feldbefunden zu holozänen Wasserspiegelschwankungen. Demgegenüber besitzen Deutungen der Seebeckenanlage und -umformung, also der hochweichselglazialen bis frühholozänen Seebildung, bislang nur spekulatives Niveau. Abgeleitet vom geologischen Bau und dem subaquatischen bzw. subaerischen Relief entwarf GEINITZ $(1886,1913)$ einen „Kombinationssee", d.h. er verwies auf die Existenz z.B. von Gletscherzungenbecken-, Rinnen-, Toteis- und Evorsionsseen unter einem Wasserspiegel. Später postulierten MARTENS (1955) einen „Sammelstausee“, DePPE \& PRIL (1958) einen rinnendurchzogenen „Endmoränenstausee“ und RÜHBERG in MÜLlER (1999) einen „Toteissee“ mit rinnenartigen Strukturen. Diese sich auf allgemeine geo- 
morphologisch-geologische Kriterien stützenden genetischen Seeklassifikationen besitzen keinen Befund-Rückhalt in Spezialkartierungen großen Maßstabs, Profilanalysen etc. Eine notwendige zukünftige Aufgabenstellung betrifft daher Untersuchungen zur Beckenstratigraphie für den Zeitraum Prä-Weichsel bis Holozän zur Beantwortung folgender Fragen: Ist das Müritz-Becken eine Bildung des Weichselglazials, oder lassen sich ältere Vorgängerbecken finden? Welche Gletscher- und Entwässerungsdynamik läßt sich für die vermutlich prägende Phase vom Frankfurter Vorstoß bis zum zweiten Pommerschen Vorstoß rekonstruieren? Welche Rolle spielte Toteis im Müritzbecken und wann taute dieses aus? Zusammen mit einer geologisch-geomorphologischen Spezialkartierung wäre dann erst die sichere Ableitung eines genetischen Seetyps möglich (vgl. MARCINEK et al. 1996). ne der Ufergestalt wie fossile Kliffs, Terrassentreppen und Strandwälle sowie Besonderheiten der archäologischen Überlieferung damit erklärt werden konnten.

In KAISER (1996a, 1998) und KAISER et al. (2000) wurden neue paläohydrologische Befunde für die Müritz vorgestellt, gemeinsam mit dem älteren Material diskutiert und ein Modell zur holozänen Seespiegel- und damit Uferlinienentwicklung entworfen (Abb. 2). Nach hohen Wasserständen von mehr als $66,5 \mathrm{~m} \mathrm{NN}$ im Weichselhochglazial zwischen Frankfurter und Pommerscher Phase deutet sich für das Spätglazial (Alleröd, Jüngere Dryas) ein Niveau von unter $62 \mathrm{~m} \mathrm{NN}$ an. Im Präboreal lag der Seespiegel um $57 \mathrm{~m} \mathrm{NN}$ und erreichte im späten Atlantikum ca. $61 \mathrm{~m} \mathrm{NN}$. Sieht man von den noch weitgehend unbekannten Niveaus des jüngs-

\section{Paläohydrologische Ent- wicklung der Müritz}

Seen sind im regionalen (z.B. JÄGER 1987), großregionalen (z.B. Yu \& HARRISON 1995) und kontinentalen Maßstab (z.B. HARRISON et al. 1996) seit einiger Zeit von besonderem Interesse für die Paläoklimaforschung. Denn während zur Ableitung der Paläotemperaturen eine Reihe von Indikatoren wie fossile Insekten, pflanzliche Reste und Sauerstoffisotopen zur Verfügung stehen, gestaltet sich die Rekonstruktion der hygrischen Bedingungen weitaus komplizierter. Ein geeigneter Indikator stellt dabei der Wasserstand von Seen dar, wobei die Grundhypothese höhere Wasserstände mit klimatisch feuchteren Phasen und niedrigere Wasserstände mit klimatisch trockeneren Phasen verknüpft. Eine Reihe von „Randbedingungen“ wie z.B. die orohydrographische Position, der hydrologische Seetyp (vgl. JeschKe 1997) oder die Größe des Einzugsgebietes modifiziert diese einfache Relation. Auch an der Müritz lag das bisherige Augenmerk der Forschung auf der Entwicklung ihres Seespiegels, da Phänome-

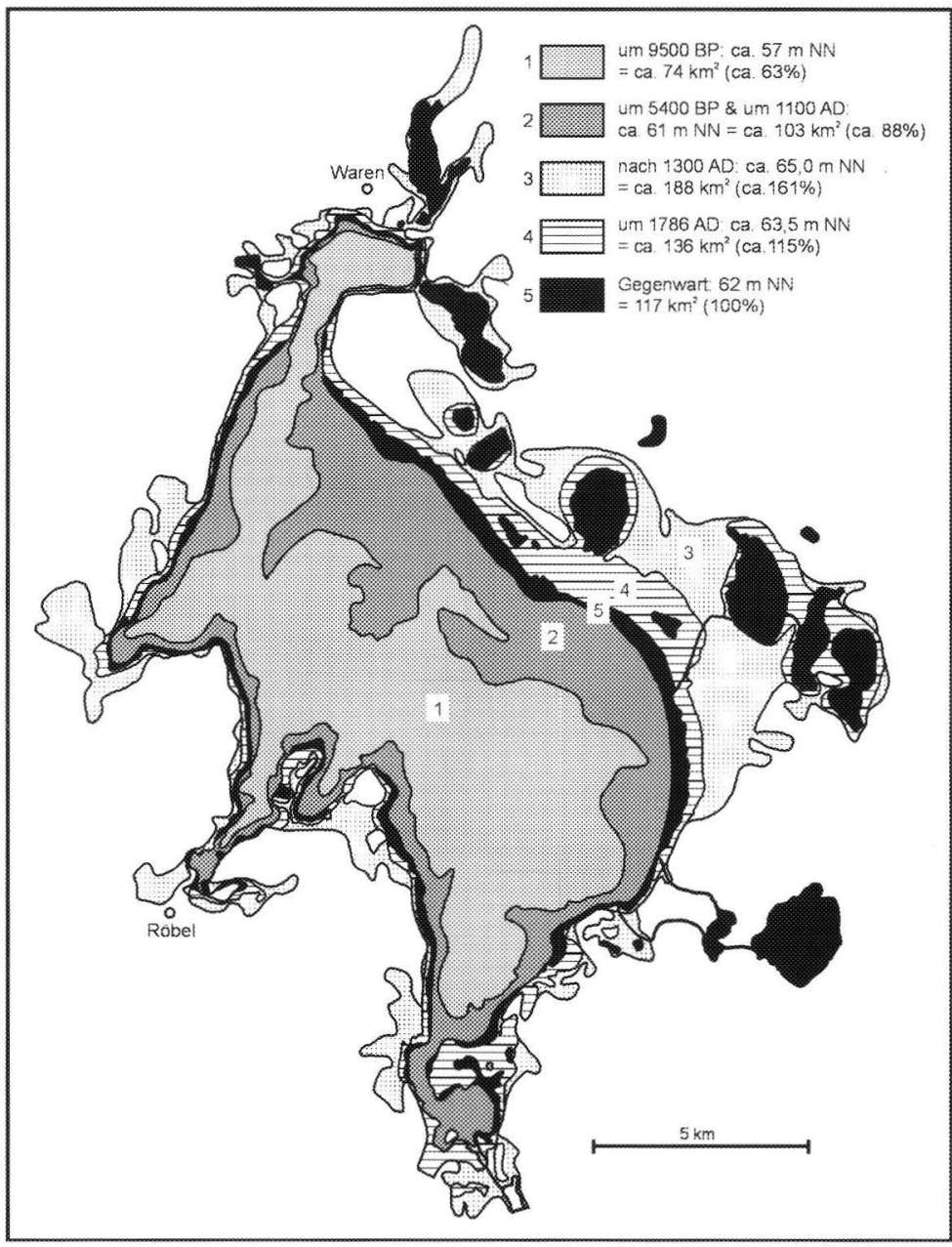

Abb. 2: Holozäne Seespiegel- und Uferlinienentwicklung der Müritz.

Fig. 2: Holocene lake-level and shore line development of Lake Mueritz. 
ten Hochglazials und des Spätglazials ab, hat sich also der See erst im Mittelholozän etwa zu den heutigen Flächen- und Volumenverhältnissen entwickelt. Nach Seespiegelschwankungen geringer Amplitude läßt sich im Subatlantikum etwa um 1100 n.Chr. ein Niveau von $61 \mathrm{~m}$ NN belegen. Mit der nachfolgenden mittelalterlich-deutschen Besiedlung sind Seespiegelanstiege auf zunächst ca. 62-63 m NN, später bis auf ca. $65 \mathrm{~m} \mathrm{NN}$ verbunden (vgl. auch RucHHÖFT 1999). Ende 18./ Anfang 19. Jh. wurde schließlich das heutige Niveau von $62 \mathrm{~m}$ NN erreicht.

Für die vormittelalterlichen Wasserstandsveränderungen sind vor allem klimatische, für die mittelund nachmittelalterlichen Seespiegelveränderun- ein größeres Seensystem, die sogenannten „Oberen Seen", zu viele Unwägbarkeiten bereithalten.

\section{Historische Bodenerosion am Ostufer der Mü- ritz}

Im Rahmen landschaftsgeschichtlicher und standortskundlicher Untersuchungen wurden Befunde erbracht, die Auskunft über die Folgen früherer Landnutzung auf Relief und Boden heute bewaldeter (aufgeforsteter) Flächen östlich der Müritz geben (KAISER 1996a, LFG M-V in Vorb.). Auf Grundlage einer Bodenkartierung im Maßstab 1:10.000 konnten entsprechende Bodenerosionsmuster für den gesamten Müritz-Nationalpark dargestellt werden (Abb. 3, vgl. DiECKManN \&

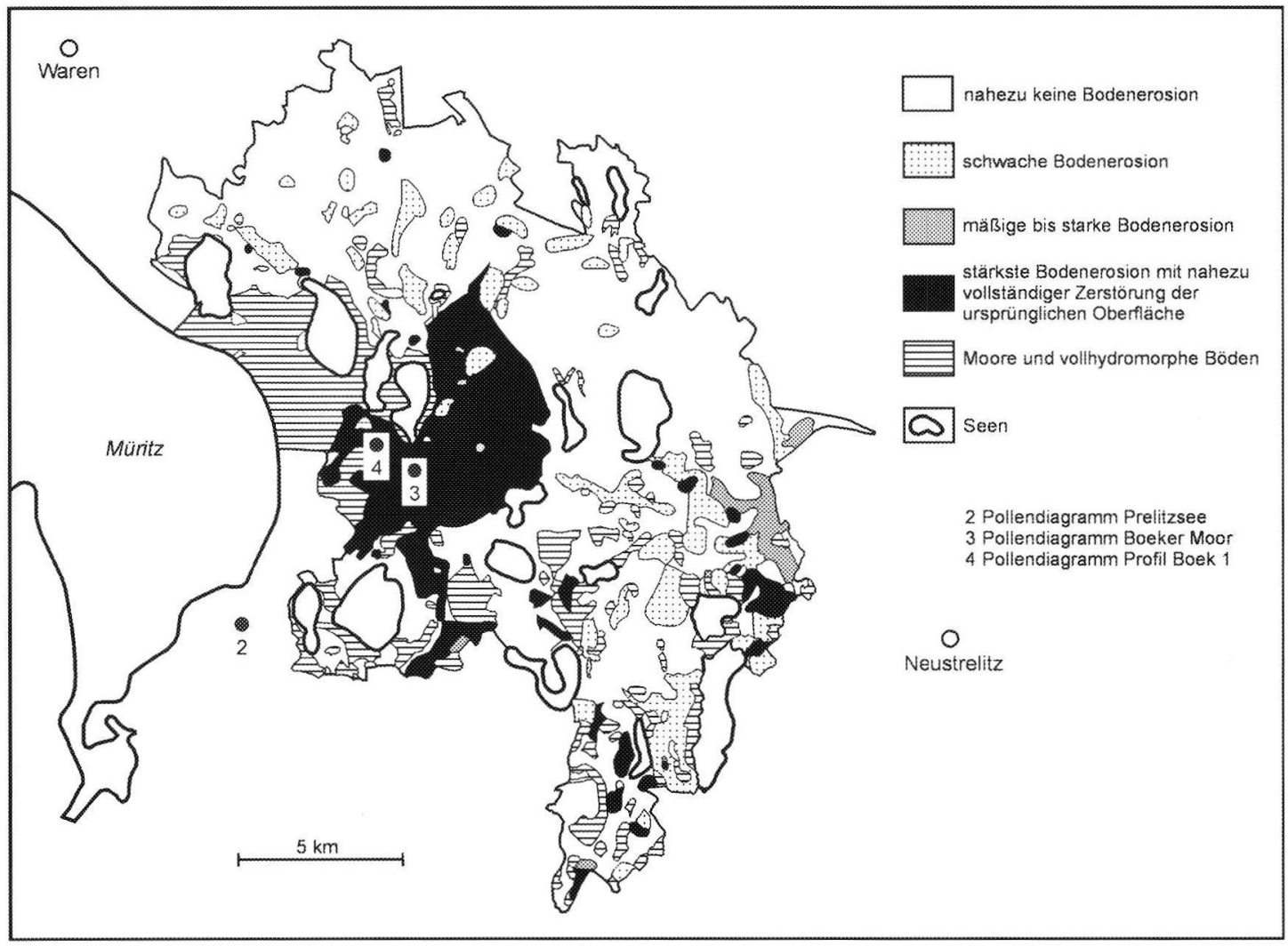

Abb. 3: Erosionsmuster im Müritz-Nationalpark, Kartierbereich Müritz.

Fig. 3: Erosion patterns in the Mueritz-Nationalpark, mapped area Mueritz.

gen anthropogene Ursachen verantwortlich. Als „kontrollierbarer“ Indikator für die paläohygrischen Verhältnisse erscheint die Müritz jedoch ungeeignet, da ihre Größe, ihr Zu- und Abfluß durch die Elde, ihr hydrogeologisch stark differenziertes Einzugsgebiet und ihre Einbindung in
KAISER 1998). Die flächenhafte Verbreitung und die Intensität der Bodenerosion nehmen dabei allgemein von den Sanderwurzeln im Norden mit abnehmender Nährkraft der Böden in Richtung der Sanderebenen und Beckensande im Süden zu. Unmittelbar östlich der Müritz fällt ein ca. $30 \mathrm{~km}^{2}$ 


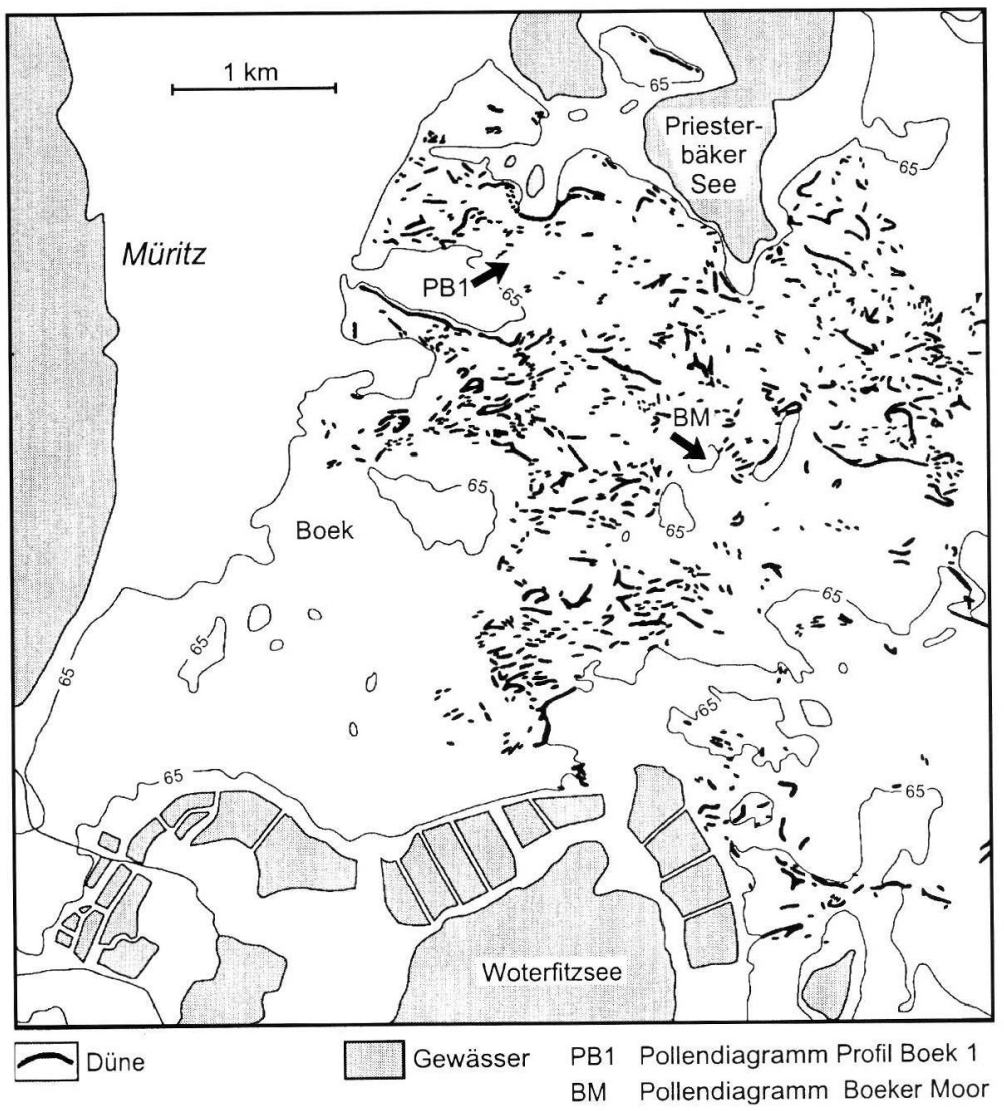

Abb. 4: Dünen am Ostufer der Müritz mit den Pollenprofilen Boeker Moor und Profil Boek 1 (Dünengrundrisse nach TK 10).

Fig. 4: Dunes at the eastcoast of Lake Mueritz and locations of the pollen diagrams Boeker Moor and Profil Boek 1.

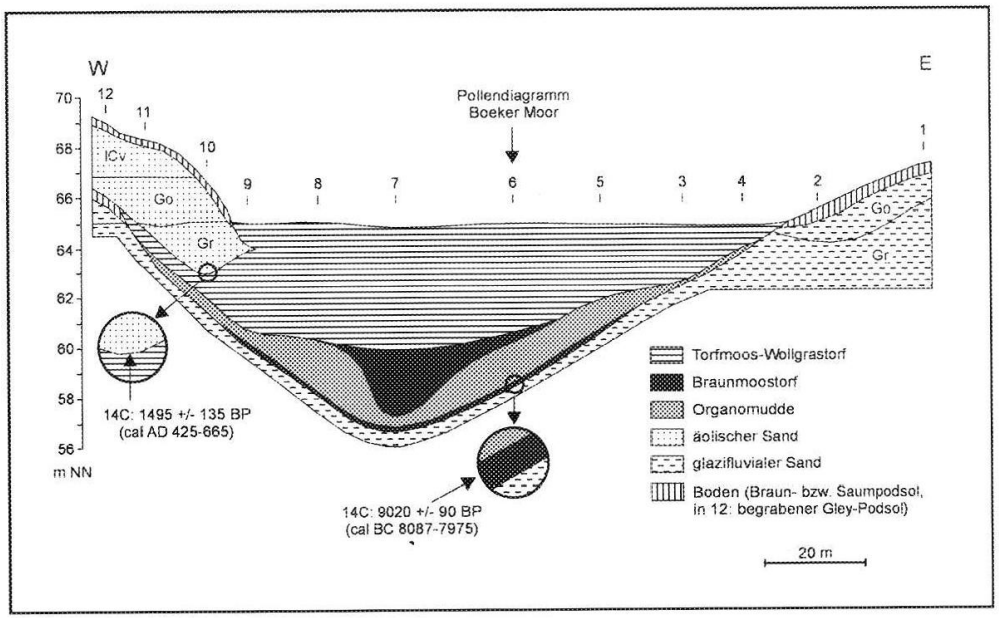

Abb. 5: Querschnitt durch das Boeker Moor.

Fig. 5: Cross section of the mire Boeker Moor. großes Areal stärkster anthropogener Überformung des ursprünglichen Boden- und Reliefinventars auf. Teile des Sanders und glazilimnischer Beckenablagerungen sind hier von Dünen und Flugsanddekken bedeckt (Abb. 4 u. 5). Auf der dem Holozän vererbten spätpleistozänen Oberfläche kamen vor dem Einsetzen der Umlagerungen großflächig Sand-Braunerden vor. Daneben existierten in geringerem Umfang Altdünen aus dem Spätglazial und dünenfreie Areale mit Sand-Podsolen sowie Sand-Gley-Podsolen. Heute sind hier als Ausdruck junger Oberflächen ausschließlich Sand-Regosole und gering entwickelte Sand-Podsole, sogenannte Sand-Saumpodsole, verbreitet. Die dominierenden Kupsten- oder Haufendünen von durchschnittlich 2 bis $4 \mathrm{~m}$, maximal $12 \mathrm{~m}$ Höhe, lassen bereits morphologisch eine anthropogen ausgelöste Genese der Dünen und Flugsanddecken vermuten. Anhand des Grundrisses in topographischen Karten zunächst als Strich- oder Längsdünen abzuleitende Formen (Abb. 4) erweisen sich häufig als gereihte Kupstendünen an Niederungsrändern. Die Mächtigkeit der Flugsanddecken beträgt 0,5 bis $2 \mathrm{~m}$.

Die in der Regel nur schwach entwickelten Böden der rezenten Oberflächen, von äolischen Sanden begrabene ehemalige Oberflächen mit stark entwickelten Böden, begrabene Moorund Seeablagerungen sowie einige mittels Radiokarbondaten, Pollenanalysen und Artefakten datierte Stratigraphien verweisen auf ein mehrphasiges Erosions-Akkumulations-Geschehen von der eisenzeitlichen oder slawischen Besiedlung 
dieses Raumes bis in das 19. Jh. Die stärksten Eingriffe sind wahrscheinlich auf das Spätmittelalter zurückzuführen, eine Reihe von Dorfwüstungen sind Zeugen dieser Nutzungsphase (vgl. Abb. 11).

\section{Palynologische Untersuchungen}

\section{Methodische Aspekte}

Die für eine Vegetationsrekonstruktion zur Verfügung stehenden Pollendiagramme Stinthorst, Prelitzsee, Boeker Moor und Profil Boek 1 stammen aus unterschiedlichen Teillandschaften des Müritzgebietes (Tab. 1 u. Abb. 1) und dienten ursprünglich verschiedenen Zwecken. Daraus resultieren Unterschiede im Probenabstand und damit in der zeitlichen Auflösung sowie in der Pollensumme pro Probe. Das am intensivsten bearbeitete Diagramm Stinthorst war vordergründig siedlungs- und vegetationsgeschichtlich orientiert (vgl. SCHOKNECHT 1996), das Diagramm Prelitzsee diente der stratigraphischen Einbindung einer limnischen Schichtenfolge (vgl. JANKE in KaISER 1996a, 1998) und das Diagramm Boeker Moor war auf einen Überblick zur lokalen Moor- und Vegetationsentwicklung gerichtet (vgl. KLoss in KAISER 1996a). Diese drei Diagramme werden nachfolgend für eine vergleichende Betrachtung der Vegetationsentwicklung im Müritzgebiet herangezogen. Das aus einem von Dünensanden begrabenen Boden erstellte Pollendiagramm Profil Boek 1 wird weiter unten gesondert vorgestellt.

Die Prozentwerte beziehen sich auf die Pollensumme aller terrestrischen Taxa (Gesamtdiagramme). In einer ersten Darstellung wurden die Spektren ihrer Tiefe in $\mathrm{cm}$ zugeordnet (Abb. 6, $7 \mathrm{u}$. 8), in einer zweiten wurde die Probenlage weniger ausgewählter Taxa auf die Zeitachse interpoliert (Abb. 9). Die Grenzen der Chronozonen III bis X (vgl. Tab. 1) wurden wie in Schoknecht (1996), MANGERUd et al. (1974) folgend, auf entsprechende ${ }^{14} \mathrm{C}$-Jahre BP für die Abschnitte nach FIRBAS (1949) als vereinfachte Berechnungsgrundlage festgelegt. Mit diesem einheitlichen Zeitmaßstab soll eine bessere Vergleichbarkeit der Diagramme erzielt werden.

Tab. 1: Erläuterungen zu den Pollendiagrammen (Abb. 6-10).

Tab. 1: Explanations to the pollen diagrams (figs. 6-10).

\begin{tabular}{|c|c|c|c|c|}
\hline Pollendiagramm & Landschaftstyp & Ablagerungsmilieu & weitere Untersuchungen & Autor und Quelle \\
\hline Stinthorst & Kames und Becken & Verlandungsmoor & - & Schoknecht 1996 \\
\hline Prelitzsee & Grundmoräne & See mit Verlandungsmoor & $\begin{array}{l}\text { Sedimentanalyse, } \\
\text { Diatomeenanalyse }\end{array}$ & $\begin{array}{l}\text { Janke in Kaiser 1996a, } \\
1998\end{array}$ \\
\hline Boeker Moor & Sander & $\begin{array}{l}\text { Versumpfungsmoor } \\
\text { über Verlandungsmoor } \\
\text { über Versumpfungsmoor }\end{array}$ & $\begin{array}{l}\text { Makrorestanalyse, } \\
\text { Radiokarbonanalyse (2) }\end{array}$ & Kloss in Kaiser 1996a \\
\hline Profil Boek 1 & $\begin{array}{l}\text { Übergang Sander } \\
\text { zu Becken }\end{array}$ & $\begin{array}{l}\text { von Flugsand } \\
\text { begrabener Boden }\end{array}$ & $\begin{array}{l}\text { Bodenanalyse } \\
\text { Radiokarbonanalyse (1) }\end{array}$ & $\begin{array}{l}\text { Kloss in Kaiser 1996a, } \\
\text { Dieckmann \& Kaiser } 1998\end{array}$ \\
\hline \multicolumn{2}{|c|}{$\begin{array}{l}\text { Grenze von Chronozonen und } \\
\text { Abschnitte nach Firbas }\end{array}$} & \multicolumn{3}{|c|}{ pollenanalytische Abgrenzungskriterien } \\
\hline \multicolumn{2}{|c|}{$\begin{array}{l}\text { Jüngere Dryas / Präboreal } \\
\text { III / IV }\end{array}$} & \multicolumn{3}{|c|}{ steiler Abfall der Nichtbaumpollen, Anstieg von Pinus bzw. Betula und Pinus } \\
\hline \multicolumn{2}{|l|}{$\begin{array}{l}\text { Präboreal / Boreal } \\
\text { IV / V }\end{array}$} & \multicolumn{3}{|c|}{ steiler Anstieg von Corylus, Einsetzen der Kurven von Quercus und Ulmus } \\
\hline \multicolumn{2}{|l|}{$\begin{array}{l}\text { Boreal / Atlantikum } \\
\text { V / VI-VII }\end{array}$} & \multicolumn{3}{|c|}{$\begin{array}{l}\text { Anstieg von Alnus, Abfall vom Corylus } \text {-Hauptgipfel, } \\
\text { letzteres z.T. erst im Atlantikum }\end{array}$} \\
\hline \multicolumn{2}{|c|}{$\begin{array}{l}\text { Atlantikum / Subboreal } \\
\text { VII / VIII } \\
\end{array}$} & \multicolumn{3}{|c|}{$\begin{array}{l}\text { klassischer Ulmenfall, Einsetzen von Siedlungszeigern, } \\
\text { Beginn der Fagus -Ausbreitung }\end{array}$} \\
\hline \multicolumn{2}{|c|}{$\begin{array}{l}\text { Subboreal / Subatlantikum I } \\
\text { VIII / IX }\end{array}$} & \multicolumn{3}{|c|}{ Anstieg der Fagus - und Carpinus -Kurven, Abfall vom letzten Corylus -Gipfel } \\
\hline \multicolumn{2}{|c|}{$\begin{array}{l}\text { Subatlantikum I / Subatlantikum IIa } \\
\text { IX / Xa }\end{array}$} & \multicolumn{3}{|c|}{$\begin{array}{l}\text { Beginn des Anstiegs zu den Maxima von Fagus und Carpinus, völkerwande- } \\
\text { rungszeitliche Siedlungslücke, Anstieg der Siedlungszeiger in slawischer Zeit }\end{array}$} \\
\hline \multicolumn{2}{|c|}{$\begin{array}{l}\text { Subatlantikum IIa / Subatlantikum IIb } \\
\mathrm{Xa} / \mathrm{Xb}\end{array}$} & \multicolumn{3}{|c|}{$\begin{array}{l}\text { steiler frühdeutscher Anstieg der Siedlungszeiger, Pinusanstieg, } \\
\text { Fagus - und Carpinus -Rückgang }\end{array}$} \\
\hline
\end{tabular}




\section{Vegetationsentwicklung im Müritzgebiet}

Die Jüngere Dryas (III) wurde mit drei Spektren an der Stinthorst erfaßt. Sie ist hier durch Maxima von Artemisia (Beifuß), Ericaceae (Heidekrautgewächse) und Cistaceae (Cistrosengewächse, hier Helianthemum, Sonnenröschen) gekennzeichnet. Die Zusammensetzung des ältesten Spektrums vom Prelitzsee mit Salix (Weide) und Juniperus (Wacholder) spricht ebenfalls für die ausklingende Jüngere Dryas.

Das Präboreal (IV) ist in allen drei Diagrammen belegt. Der Anteil von Pinus (Kiefer) und Betula (Birke) ist standörtlich verschieden. Im Boeker Moor wurde der Übergang zum Boreal durch ein ${ }^{14} \mathrm{C}-\mathrm{Da}$ tum von $9020 \pm 90 \mathrm{BP}(\mathrm{Hv}-19535)$ an Kiefernholz in Braunmoostorf erfaßt (Abb. 5). Da die Datierung jedoch an einer neben dem Pollenprofil niedergebrachten Nachbohrung erfolgte, kann keine exakte Projektion des Datums in das Pollendiagramm vorgenommen werden.

Im Boreal $(V)$ entwickelte sich die Vegetation in den Gebieten an der Stinthorst und am Boeker Moor ähnlich. Es ist ein rasanter Rückgang von Pinus bei gleichbleibenden oder leicht ansteigenden Anteilen von Betula zu beobachten. Im Gegensatz dazu nahmen am Prelitzsee Kiefer und Birke gleichermaßen allmählich ab. Corylus (Hasel) breitete sich rasch aus und war praktisch für das ganze Boreal vegetationsprägend. Das Einsetzen und der rasche Anstieg der Kurven von Tilia (Linde) und Alnus (Erle) kennzeichnen den Übergang zum Atlantikum.

Die Vegetationsentwicklung der Eichenmischwälder im Atlantikum (VI-VII) verlief recht gleichförmig. In den Anteilen der Eichenmischwaldarten Quercus (Eiche) bis Fraxinus (Esche) werden Unterschiede zwischen den Untersuchungspunkten deutlich. Um den Prelitzsee spielte vor allem Quercus eine größere Rolle als in den anderen beiden Gebieten. Im Diagramm Stinthorst haben Tilia und Ulmus (Ulme) im Verlauf des Subboreals (VIII) eine kurze Wiederausbreitungsphase ähnlich anderen Untersuchungspunkten westlich der Müritz (SCHOKNECHT 1996). In den beiden Gebieten östlich der Müritz ist eine solche Phase nicht zu erkennen. Die Ausbreitung von Fagus (Rotbuche) erfolgte an den drei Untersuchungspunkten allmählich, die Kurven erreichen zum Ende dieses Abschnittes knapp $1 \%$. Pollen von Carpinus (Hainbuche) treten an der Stinthorst nur vereinzelt auf, die Kurve schließt sich erst im Subatlantikum. Am Boeker Moor und am Prelitzsee hingegen ist die Kurve von Carpinus schon im Subboreal geschlossen.

Am Prelitzsee läßt sich etwa seit Beginn des Subboreals Landnutzung erkennen, die offenbar in der Bronzezeit ihren Höhepunkt erreichte: Quercus ging kräftig zugunsten von Pinus zurück. Juniperus, Chenopodiaceae und Poaceae breiteten sich aus. Ähnlich, aber schwächer ausgeprägt, verlief die Entwicklung am Boeker Moor. An der Stinthorst erreicht die Summe der Siedlungszeiger kaum mehr als 5 $\%$. Eine mittelneolithische Siedlungsphase wird durch Plantago lanceolata (Spitzwegerich) markiert. Erst für die Bronzezeit kann Cerealia-Pollen (Getreide) nachgewiesen werden.

Das Subatlantikum I (IX) ist die Periode, die durch den Anstieg der Kurven von Fagus und Carpinus zu ihrem Hauptmaximum gekennzeichnet ist. In vielen Diagrammen der weiteren Region läßt sich in dieser Zeit ein erstes Plateau erkennen (z.B. MÚlLER \& KoHL 1966, Kloss 1980). Im Untersuchungsgebiet ist dieser Verlauf in unterschiedlicher Ausprägung ebenfalls zu identifizieren. An der Stinthorst finden wir in dieser Zeit hohe Anteile von Pinus. Fagus und Carpinus spielten mit weniger als $2 \%$ bzw. $1 \%$ nur eine untergeordnete Rolle. Der Anteil von Quercus veränderte sich kaum und liegt mit $10 \%$ etwa so hoch wie in den anderen beiden Diagrammen. Auffällig ist im Diagramm Stinthorst die Zunahme von Cyperaceen und Poaceen (Sauer- und Süßgräser). In Verbindung mit dem kurz vorher erfolgten Umschlag von Mudde- zu Torfsedimentation spiegelt sich hier die lokale Gewässerverlandung wider. Siedlungszeiger im engeren Sinne spielen kaum eine Rolle. Im Zusammenhang mit den Gehölzkurven ergeben sich weitere Folgerungen. Pinus als stark windblütiger Baum mit hoher Pollenproduktion ist in Zeiten der Waldauflichtung überrepräsentiert. Quercus reagiert gering auf extensiven Weideeinfluß, Rodungen hingegen werden von der Quercus-Kurve deutlich reflektiert. Fagus reagiert als älterer Baum bedeutend empfindlicher auf Verbiß und Schälschäden. Als junges Gehölz ist sie jedoch sehr regenerativ. Carpinus ist auch als älteres Gehölz noch sehr regenerationsfähig. Fagus und Carpinus können als Neuankömmlinge im Gebiet um die Stinthorst durch hohen Verbißdruck am Erreichen der Blühfähigkeit gehindert worden sein. Sie sind deshalb nur mit wenigen Prozenten im Pollendiagramm vertreten. Das Gebiet um die Stinthorst mit seinen von Mooren und Gewässern umgebenen Sandkuppen wurde vermutlich vorrangig beweidet; Ackerbau in Verbindung mit Rodung fand zu dieser Zeit nicht statt. Am Boeker Moor zeigen Fagus und Carpinus einerseits und Pinus andererseits gegenläufige Entwicklungen. Carpinus und Fagus erreichen um bzw. über $10 \%, P i$ nus fällt von etwa $45 \%$ auf $10 \%$ ab. Die QuercusKurve bleibt von diesem Geschehen unberührt. 


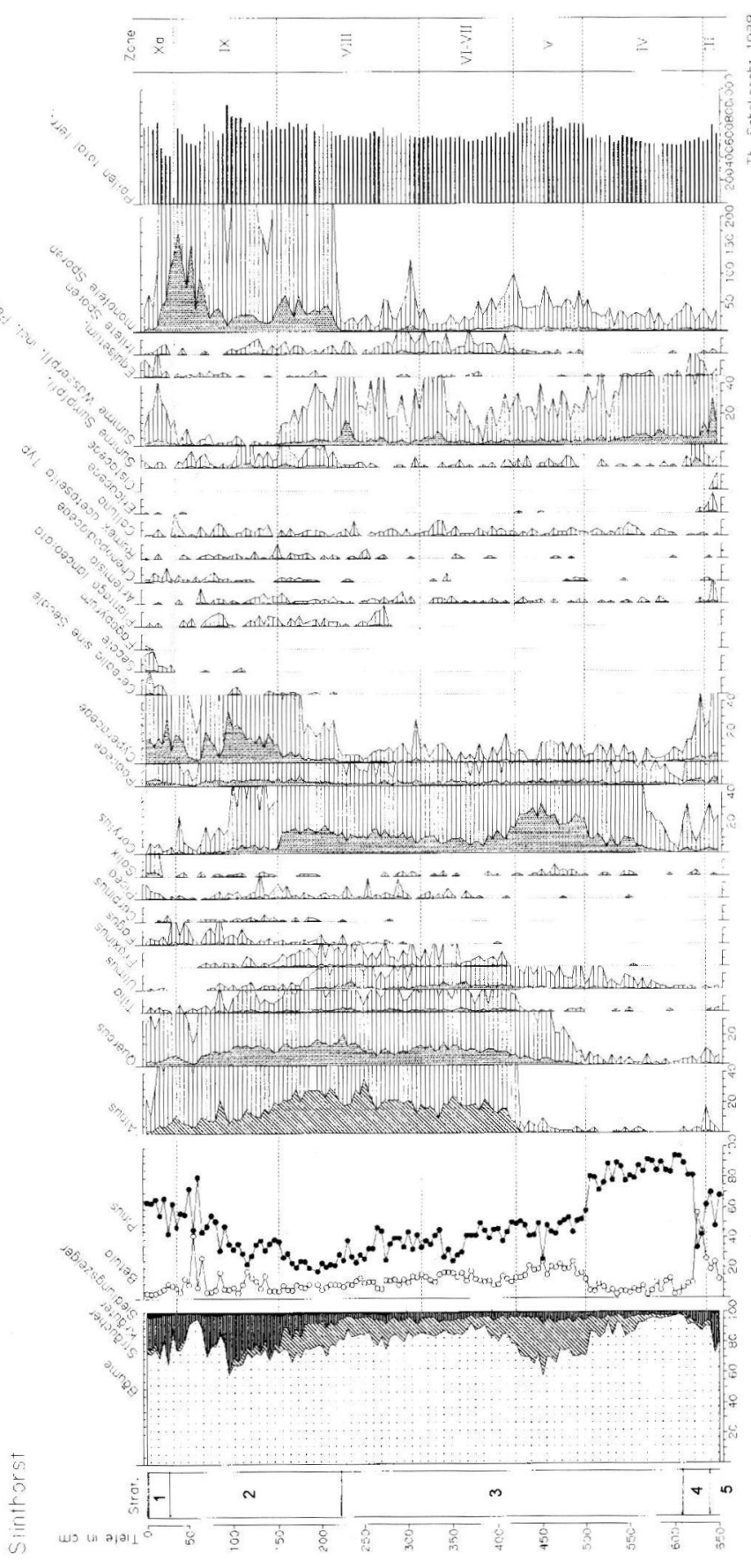

Abb. 6: Pollendiagramm Stinthorst, Gesamtdiagramm. Ausgewählte Taxa. Lithostratigraphie: 1 Bruchwaldtorf, 2 Riedtorf, 3 Kalkmudde, 4 Torfmudde, 5 Sandmudde.

Fig. 6: Pollen diagram Stinthorst, total diagram. Selected taxa. Lithostratigraphy: 1 wood peat, 2 radicel peat, 3 calcareous gyttja, 4 peaty gyttja, 5 sandy gyttja.
Der Verlauf der Siedlungszeigerund übrigen Kräuterkurven am Boeker Moor ist relativ flach und unspezifisch. Plantago lanceolata und Artemisia haben geschlossene Kurven, andere Taxa treten dagegen nur mit einzelnen Pollenkörnern auf. Siedlungsplätze mit Äckern und Weideland dürften in einigen Kilometern Entfernung gelegen haben. Eine völkerwanderungszeitliche Siedlungslücke zeichnet sich in allen drei Diagrammen deutlich ab. Der westliche Rand des Boeker Moores wird von einer in das Moor gewanderten Düne gebildet. Ein ${ }^{14} \mathrm{C}$-Datum von $1495 \pm$

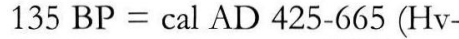
22342) an Torfmoos-Wollgrastorf unmittelbar unter dem Dünensand könnte ein völkerwanderungszeitliches bis frühslawisches Erosions- bzw. Nutzungsereignis widerspiegeln, stände dem nicht der Pollenbefund entgegen. Ein paralleles Pollenspektrum aus dem datierten Torf spricht nach Korrelation mit dem Pollendiagramm Boeker Moor eher für eine Datierung in das Jüngere Subatlantikum $(\mathrm{Xa} / \mathrm{Xb})$. Am Prelitzsee erreicht die FagusKurve ein Plateau von ca. $5 \%$, die Carpinus-Kurve nur 1-2\%. Entsprechend höher bleiben mit $30 \%$ die Pinus-Werte. In diesem Diagramm sind zwei deutliche Siedlungsphasen zu erkennen (ca. 370-340 cm): Die Summenkurven von Kräutern und Siedlungszeigern laufen parallel. Wichtige Taxa unter den Siedlungszeigern sind Plantago lancelata, Artemisia und Rumex acetosella (Kleiner Ampfer), in der jüngeren Siedlungsphase kommen Cerealia hinzu. Offenbar haben während der Vorrömischen Eisenzeit und der Römischen Kaiserzeit in der Nähe Siedlungen gelegen, jedoch nicht in unmittelbarer Nachbarschaft zum See. Im Vergleich mit dem Boeker 


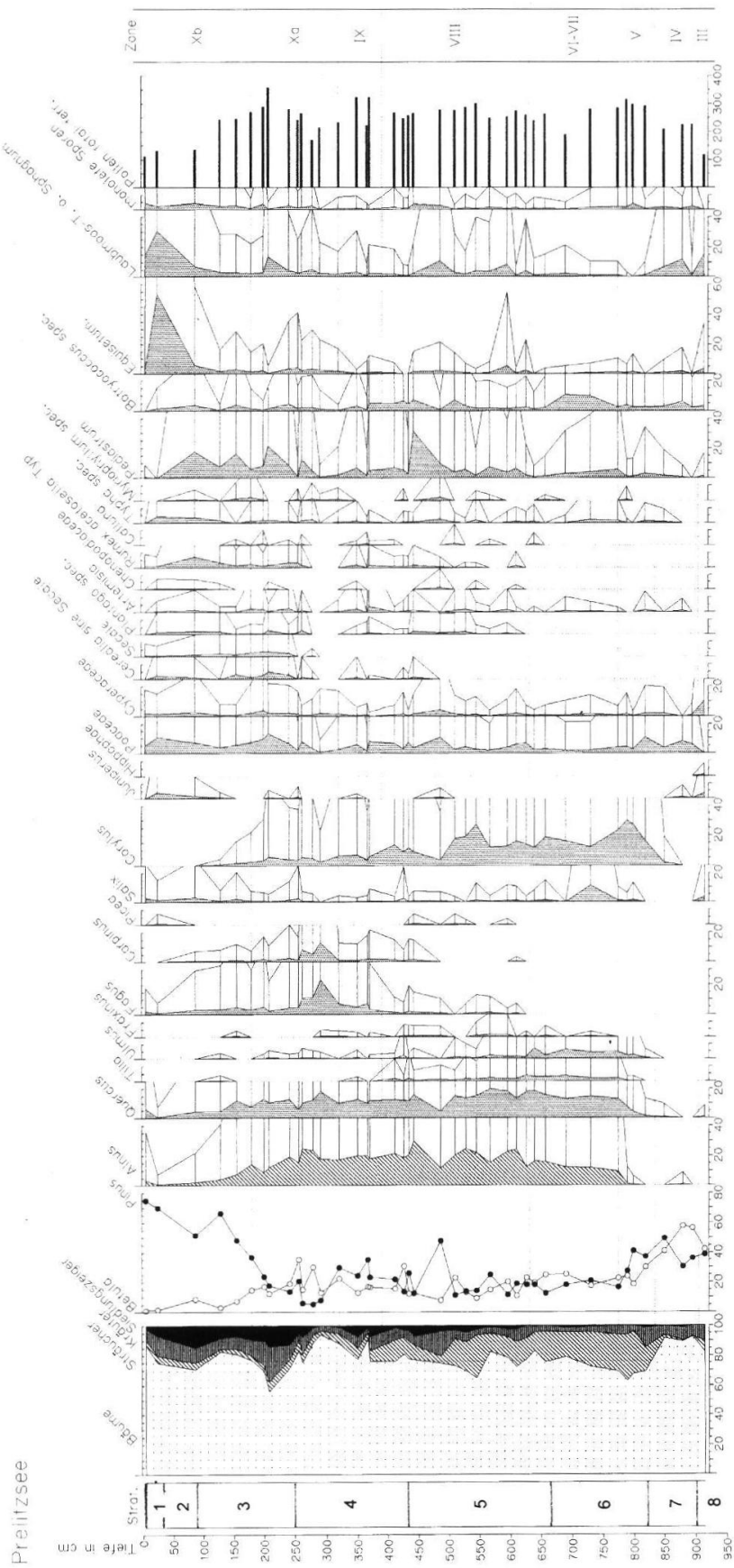

Abb. 7: Pollendiagramm Prelitzsee, Gesamtdiagramm. Ausgewählte Taxa. Lithostratigraphie: 1 Schilftorf, 2 Wasser, 3 Kalkmudde, 4 Wechsellagerung von Kalkmudde und Organomudde, 5 Organomudde, 6 Wechsellagerung von Kalkmudde und Organomudde, 7 Schluffmudde, 8 Sandmudde.

Fig. 7: Pollen diagram Prelitzsee, total diagram. Selected taxa. Lithostratigraphy: 1 P bragmites peat, 2 water, 3 calcareous gyttja, 4 alternating calcareous gyttja and organic gyttja, 5 organic gyttja, 6 alternating calcareous gyttja and organic gyttja, 7 silty gyttja, 8 sandy gyttja.
Moor wird deutlich, daß die Ausbreitung von Fagus und Carpinus am Prelitzsee durch anthropogenen Einfluß unterdrückt wurde. Ein kleiner Fagus-Gipfel in der Zeit der Siedlungsruhe verstärkt dieses Bild.

Die jüngsten Spektren des Diagramms Stinthorst sind dem Beginn des Subatlantikums II (Xa) zuzuordnen. Dafür spricht vor allem das Einsetzen der geschlossenen Secale-Kurve. Die wenigen Spektren sind jedoch für das weitere vegetations- und siedlungsgeschichtliche Geschehen nicht auswertbar. Am Boeker Moor bleiben die relativ hohen Fagus-Werte aus dem Subatlantikum I erhalten, Carpinus erreicht ihren Maximalwert und Pinus bleibt im Minimum. Mit dem Wiedereinsetzen bzw. der Zunahme der Siedlungszeigerwerte und dem Beginn von Secale fallen die Fagusund Carpinus-Kurve von ihrem Hauptgipfel. Eine Intensivierung des Siedlungsgeschehens in spätslawischer Zeit bewirkte dann auch einen Abfall der Kurve. Spätestens mit der mittelalterlich-deutschen Besiedlung dieses Gebietes verloren die Laubgehölze an Bedeutung. Pinus wird in den Diagrammen einerseits durch die Öffnung der Landschaft überrepräsentiert, andererseits wurde sie durch selektive Holzentnahme und ihr hohes Sukzessionspotential auf Brachflächen anthropogen gefördert. Ein Wechsel der Siedlungszeiger bzw. kulturbegleitenden Arten wie Calluna (Heidekraut) und Artemisia zu Poaceen bei unveränderten Getreidewerten ist möglicherweise der Ausdruck für eine veränderte Wirtschaftsweise: Extensive Beweidung von Wäldern und Brachen wurde durch flächenhafte Grünlandnutzung abgelöst. Am Prelitzsee ist die Waldentwicklung mit der Situation am Boeker Moor vergleichbar. Fagus und Carpinus erreichten Maxima, auch Quercus breitete sich noch einmal aus. Ausbreitung als auch nachfolgender Rückgang waren wie am Boeker Moor das Ergebnis der völkerwanderungszeitlichen Siedlungslücke bzw. der anschließenden slawischen Besiedlung. Der Nutzungseinfluß ist am Prelitzsee kräftiger als am Boeker Moor ausge- 


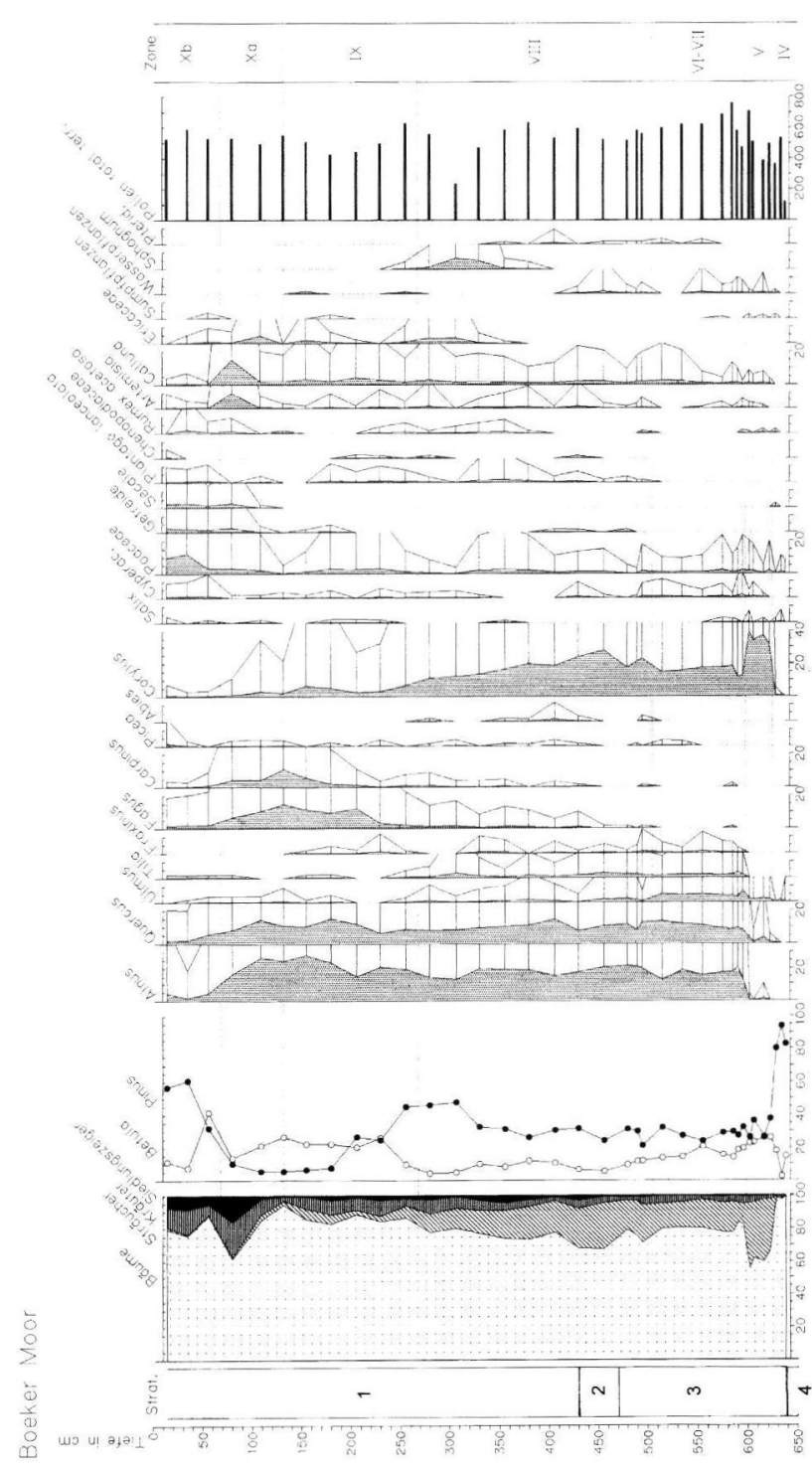

Abb. 8: Pollendiagramm Boeker Moor, Gesamtdiagramm. Ausgewählte Taxa. Lithostratigraphie: 1 Torfmoos-Wollgrastorf, 2 Braunmoostorf, 3 Organomudde, 4 Feinsand.

Fig. 8: Pollen diagram Boeker Moor, total diagram. Selected taxa. Lithostratigraphy: 1 Sphagnum-Eriophorum peat, 2 brownmoss peat, 3 organic gyttja, 4 fine sand.

prägt. Das gilt auch für den jüngsten Teil des Subatlantikums II (Xb). Hier macht sich die extensive Waldnutzung (Waldweide) durch die Wiederausbreitung von Juniperus bemerkbar, allerdings ohne $\mathrm{da}$ es zu einer Verheidung durch Calluna kommt. Einen vergleichenden Überblick zur holozänen Entwicklung der Waldvegetation und des anthro- pogenen Einflusses in den drei Diagrammen vermittelt Abbildung 12.

\section{Pollendiagramm Profil Boek 1}

Dieses Diagramm (Abb. 10; vgl. DiECKMANN \& KAISER 1998) stammt aus einem von Flugsanden begrabenen Gley-Eisenhumuspodsol, der sich in Beckensanden entwickelt hat. Ziel der Pollenanalyse des in Zentimeterabständen lückenlos untersuchten Profils war eine Datierung des Überdeckungszeitpunktes, also des Beginns äolischer Aktivität vor Ort, sowie die Rekonstruktion der lokalen Vegetation vor der Überdeckung (zur Lage vgl. Abb. 4).

Bodenpollenanalysen haben sich als ein wertvolles Mittel zur Rekonstruktion der lokalen Vegetationsentwicklung bis zu einer Entfernung von 20-30 m um den Untersuchungspunkt erwiesen (ANDERSEN 1986, 1998). Der gegenüber Mooroder Seeprofilen andersartige Pollenniederschlag und vertikale Pollentransport durch Bioturbation und Perkolation ist bei der Interpretation solcher Diagramme zu beachten. Ein benachbartes Diagramm mit dem extralokalen bis regionalen Pollenniederschlag, wie in diesem Falle das Diagramm Boeker Moor, sollte zur Verfügung stehen.

Im nordostdeutschen Tiefland hat erstmals ENGMANN (1937) in größerem Umfang Bodenpollendiagramme bearbeitet. Systematische Untersuchungen von MüLLER an rezenten als auch begrabenen Humusauflagen, Podsolen, Gley-Podsolen, Braunerden und Gleyen in Mecklenburg-Vorpommern und Brandenburg zeigten eine Pollenführung in begrabenen Sand-Gley-Podsolen bis in das Jüngere Atlantikum (VII) (MülLer et al. 1971, KRETSCHMER et al. 1971).

An der Basis des Diagramms Profil Boek 1 wird der Übergang Subboreal/Subatlantikum (VIII/IX) widergespiegelt. Mit dem Auftreten bzw. der Zunahme von Fagus, Carpinus, Plantago lanceolata und anderen Siedlungszeigern sinken die Anteile von Tilia drastisch. Seitdem und bis zur stärkeren Ausbreitung von Fagus ab etwa $71 \mathrm{~cm}$ Tiefe hatten auch Pinus und Corylus hohe Anteile an der Pollenführung. Bemerkenswert ist der konstant niedrige Wert von Quercus von weniger als $5 \%$ bis zum Fagus-Anstieg. 


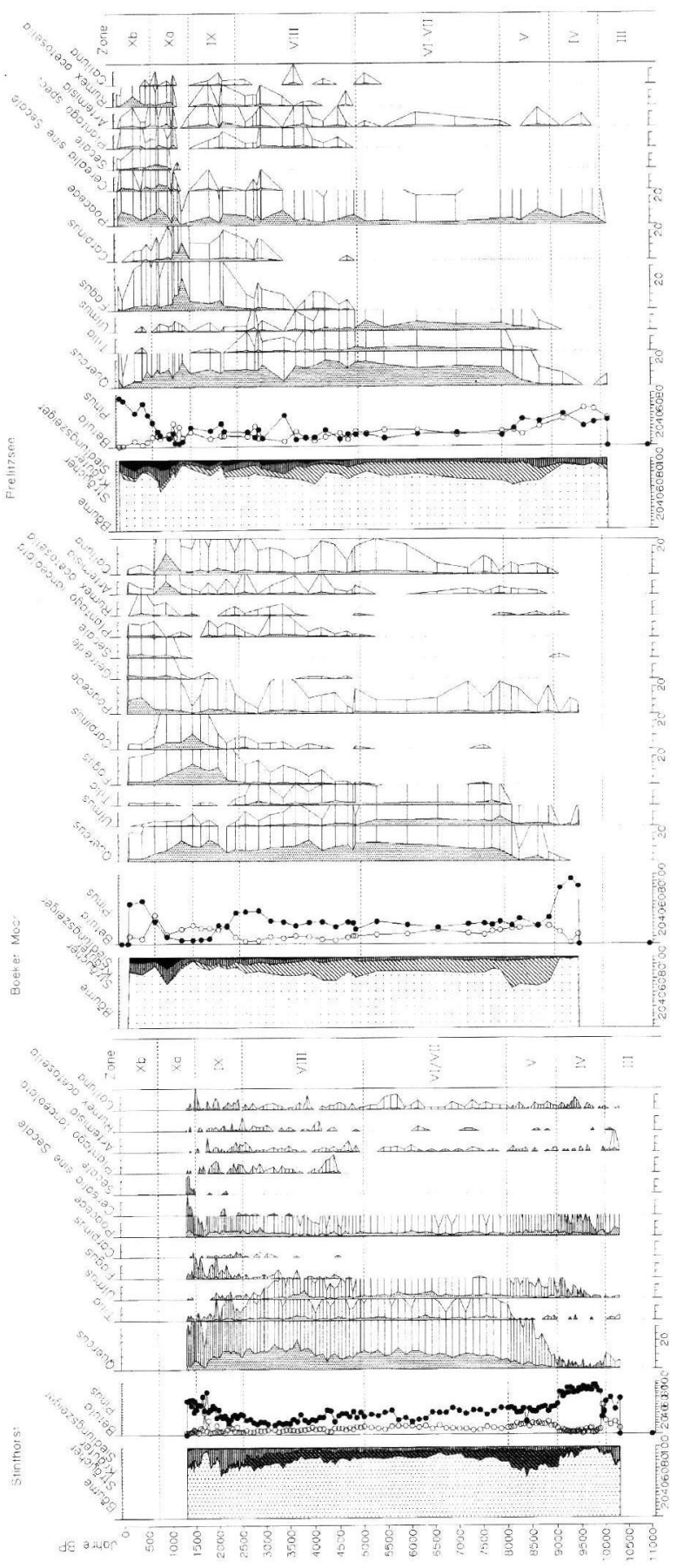

Abb. 9: Synoptische Darstellung der Pollendiagramme Stinthorst, Boeker Moor und Prelitzsee auf einer linearen Zeitskala $\left({ }^{14} \mathrm{C}\right.$-Jahre BP). Ausgewählte Taxa.

Fig. 9: Synopsis of the pollen diagrams Stinthorst, Boeker Moor and Prelitzsee on a linear time scale $\left({ }^{14} \mathrm{C}\right.$-years BP). Selected taxa.
Die jüngsten Spektren gehören zu einer Siedlungsphase, die mit dem Nachweis von SecalePollen mindestens in slawische Zeit gestellt werden kann. Auffällig sind die mit 20-40\% sehr hohen Calluna-Werte, die offenbar nicht anthropogen sind. Calluna ist in Nordostdeutschland eine lokale Komponente in der Moorvegetation in Verbindung mit der Ausbildung von Gley-Podsolen (MüLLER et al. 1971, BRANDE 1995).

Hinsichtlich der grundlegenden vegetationsgeschichtlichen Abläufe ähnelt das Diagramm Profil Boek 1 dem vom Boeker Moor. Die Unterschiede im Detail sind jedoch erheblich. Bis auf die letzten drei Spektren hat Tilia höhere Anteile als Ulmus und Quercus. Die starke Dominanz von Tilia in den unteren $5 \mathrm{~cm}$ des Diagramms verschiebt nahezu alle anderen Pollenwerte. Im Boeker Moor hat Quercus erwartungsgemäß die größeren Anteile. Auch die hohen Calluna-Werte treten im Boeker Moor nicht auf. Annähernd so hohe Werte werden hier einmalig in spätslawischer Zeit gefunden und deuten zusammen mit dem Artemisia-Gipfel auf ein Brachfallen von Äckern und die Ausweitung von Weideflächen.

Ein ${ }^{14} \mathrm{C}$-Datum an Huminsäure aus dem ersten Zentimeter des rGo-fAeh-Horizontes von Profil Boek 1 ergab $1370 \pm 60 \mathrm{BP}=$ cal AD 640-686 (Hv-19537). Dieses Datum, das in die frühslawische Siedlungsphase fällt, fügt sich zwar zwanglos in die Zeit der maximalen $\mathrm{Fa}$ gus-Werte im älteren Teil des Subatlantikums II ein (Xa, vgl. Abb. 9 u. 12). Ob damit aber auch näherungsweise der Zeitpunkt der äolischen Sandüberdeckung widergespiegelt wird, muß mangels einer breiteren ${ }^{14} \mathrm{C}$-Datenbasis offen bleiben. Neben dem unmittelbar vor der Sandüberdeckung gebildeten Humus ist mit einem unbekannt hohen Anteil an mehrhundertjährigem alten Humus im Boden zu rechnen, was eine Altersüberschätzung zur Folge haben kann (GEYH 1983).

\section{Archäologische Untersuchungen}

\section{Ur- und frühgeschichtliche Besiedlung im Umland der Müritz}

In den Jahren 1984/85 wurde eine Untersuchung zur ur- und frühgeschichtlichen Fundplatzverteilung im Umland der Müritz durchgeführt (PREHN 1985). Dabei war zu prüfen, ob und in wieweit diese einerseits durch den Forschungsstand der Bodendenkmalpflege 


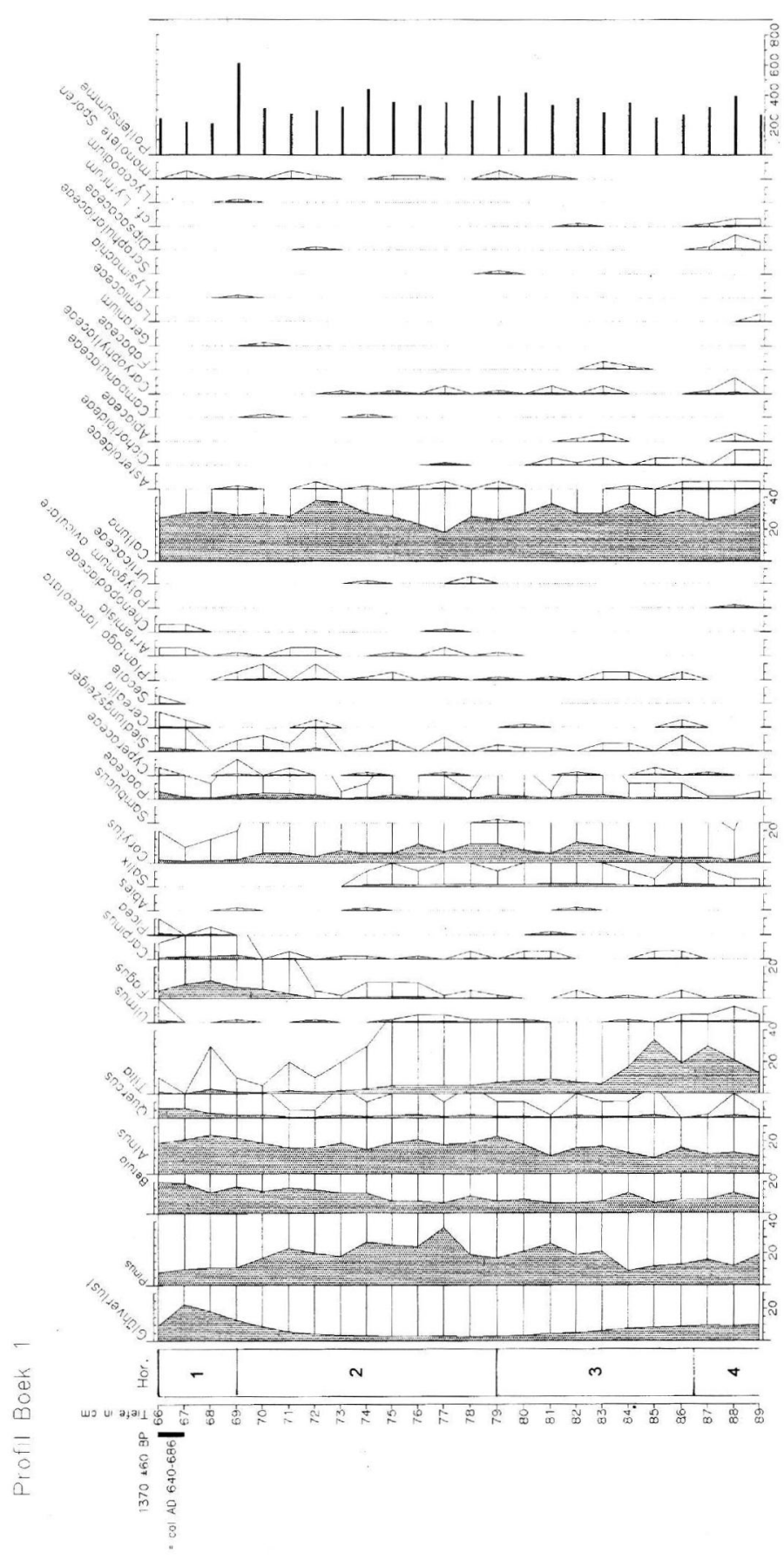

Abb. 10: Pollendiagramm Profil Boek 1, Gesamtdiagramm. Bodenhorizonte des begrabenen Gley-Eisenhumuspodsols: $1 \mathrm{rGo}-\mathrm{fAeh}, 2 \mathrm{fAhe,} 3 \mathrm{fBh}, 4 \mathrm{fBsh}$.

Fig. 10: Pollen diagram Profil Boek 1, total diagram. Soil horizons of the buried gleyic Podzol: 1 rGo-fAeh, 2 fAhe, 3 fBh, 4 fBsh. und andererseits durch verschiedene natürliche Faktoren wie die Lage zum Gewässer, das Relief und die Bodenverhältnisse beeinflußt ist. Im Rahmen einer interdisziplinären Bestandsaufnahme zur Entwicklung des Müritzgebietes im Holozän konnte der Datenbestand 1998 ergänzt werden.

Das Arbeitsgebiet (Abb. 11) umfaßt das Umland der Müritz in einem etwa uferparallelen Streifen von ca. $3 \mathrm{~km}$ im Osten und die Ufergemarkungen im Westen der Müritz. Die Fundplatzaufnahme erfolgte anhand von Gemarkungsakten; darüber hinaus konnten im begrenzten Maße Flurbegehungen durchgeführt werden. Zu berücksichtigen ist, daß das stark bewaldete und vermoorte Ostufer der Müritz auch infolge jahrzehntelanger Staatsjagdnutzung nahezu unzugänglich war und daher für eine Kartierung von Fundplätzen nahezu ausfiel. Das heißt, daß in einigen Gemarkungen die archäologischen Nachweise stark zufallsgeprägt sind. Aussagen zur Siedlungslage und -intensität lassen sich daher für bestimmte ur- und frühgeschichtliche Perioden nur sehr vorsichtig treffen.

Die Zahl der exakt lokalisierbaren Fundplätze ist mit 401 trotz der erwähnten z.T. eingeschränkten Tätigkeit der Bodendenkmalpflege relativ hoch. Dabei handelt es sich fast ausschließlich um durch Oberflächenfunde und Notbergungen ermittelte Fundplätze. Systematische Ausgrabungen außerhalb der Städte erfolgten kaum (HoLlNAGEL \& SCHOKNECHT 1956, SCHOKNECHT 1959, BLEILE 2000).

Die zeitliche Verteilung der Fundplätze im Arbeitsgebiet gestaltet sich wie folgt: Spätpaläolitikum 4, Mesolithikum 69, Neolithikum 174, Bronzezeit 84, Vorrömische Eisenzeit 41, Römische Kaiserzeit 31, Völkerwanderungszeit 1 und schließlich Slawenzeit 99. An unbestimmbaren Fundplätzen wurden 72 ermittelt. Auf verschiedenen 

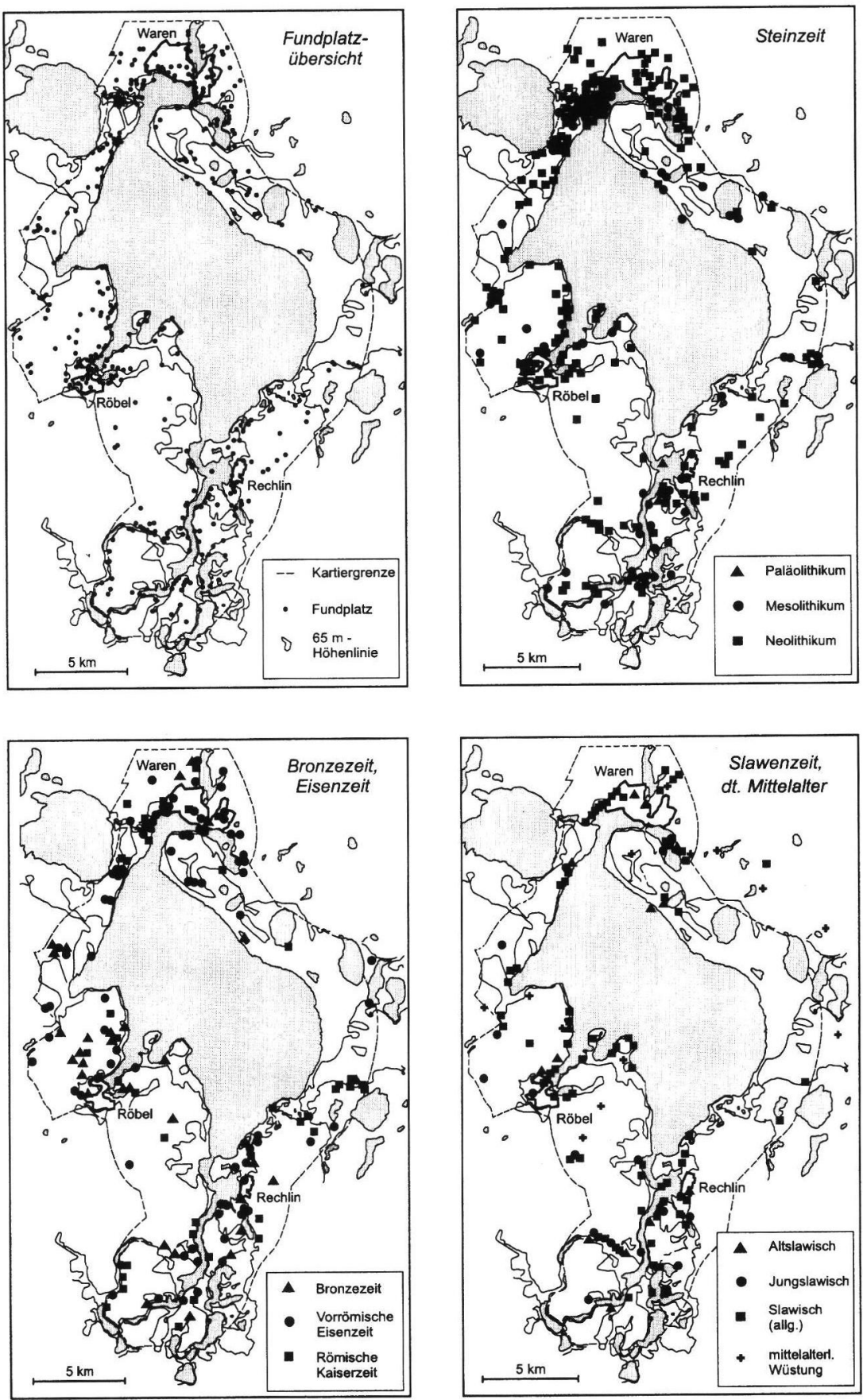

Abb. 11: Archäologische Fundplatzverteilung im Umland der Müritz.

Fig. 11: Archaeological sites around the Lake Mueritz.

Fundplätzen sind mehrere ur- und frühgeschichtliche Besiedlungsphasen feststellbar.

Deutlich zeigen sich gravierende Unterschiede im
Nachweis der ur- und frühgeschichtlichen Perioden (vgl. SCHOKNECHT in KNAPP et al. 1999). So ist das Spätpaläolithikum mit nur wenigen Fundplät- 


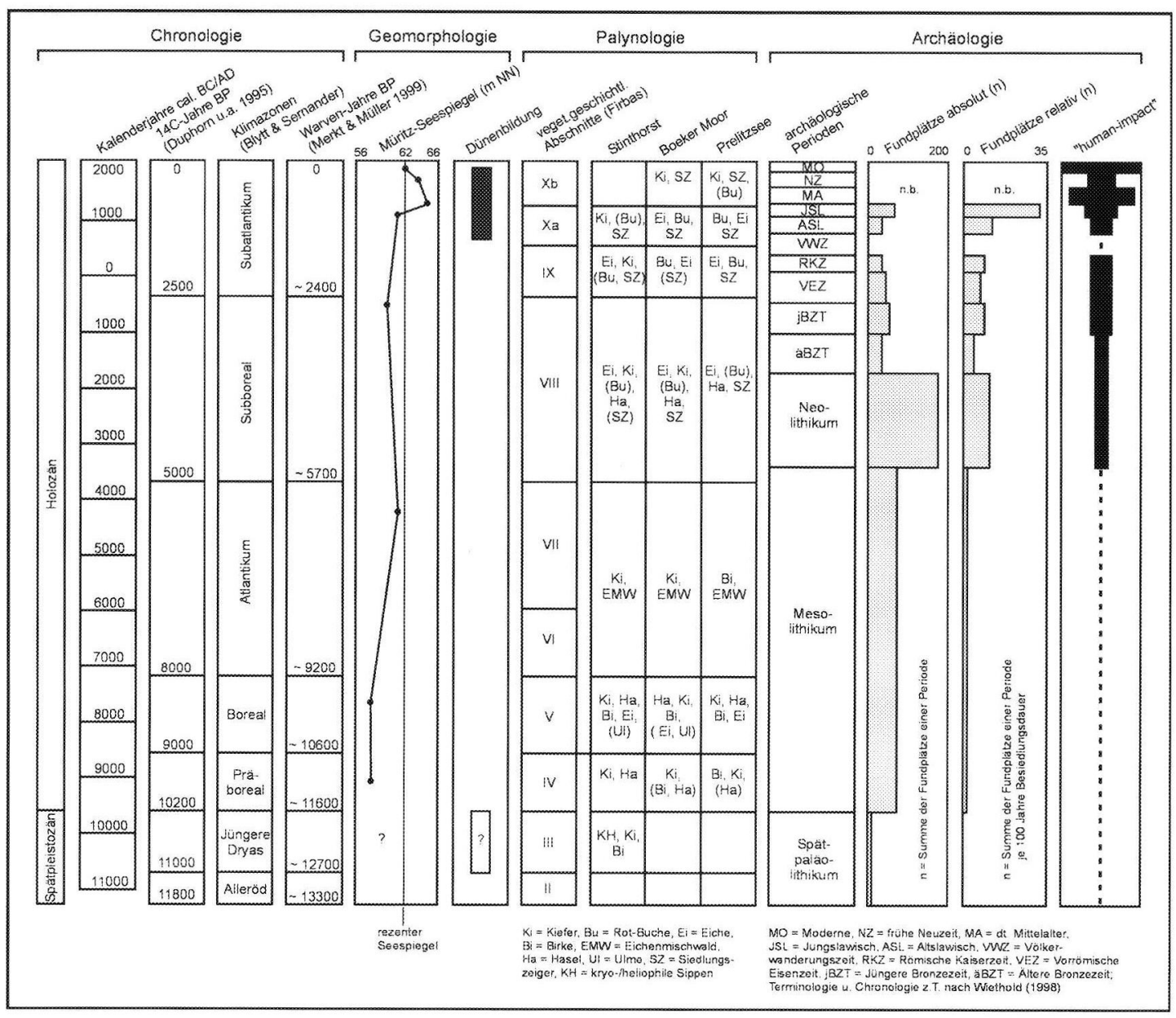

Abb. 12: Übersicht zur Landschaftsentwicklung im Müritzgebiet.

Fig. 12: Schemed landscape development in the Lake Mueritz area.

zen sogenannter Stielspitzen vor allem auf Inseln belegt. Im Mesolithikum wurden die unmittelbaren Uferlagen offener Wasserflächen bevorzugt. Besonders dicht erscheint die Besiedlung am Eldeausfluß aus der Müritz. Für das Neolithikum ist die höchste Fundplatzzahl zu verzeichnen; das Übergewicht der neolithischen Fundplätze wird jedoch durch die unterschiedliche Dauer der urund frühgeschichtlichen Perioden relativiert (Abb. 12). Zudem handelt es sich überwiegend um Einzelfunde (z.B. Steinbeile), die zumeist in unmittelbarer Gewässernähe geborgen wurden. Die wenigen Siedlungen mit Keramikfunden sind, mit Ausnahme der Insel Stinthorst (vgl. Abb. 6), vorwiegend in erhöhter Uferlage anzutreffen. Besiedlungskonzentrationen sind am Nord- und Westufer sowie im Süden erkennbar. Von den bronze- zeitlichen Fundplätzen ist der überwiegende Teil der jüngeren Bronzezeit zuzuordnen. Die entsprechenden Siedlungen wurden bevorzugt in unmittelbarer Ufernähe, jedoch in unterschiedlicher Höhenlage angelegt; eine relativ geschlossene Besiedlung deutet sich am Nordufer an. Für die Vorrömische Eisenzeit und die Römische Kaiserzeit zeichnet sich ein Besiedlungsrückgang ab. Die Siedlungen befinden sich an heutigen Niederungsrändern und kleinen Gewässern, während die Ufer der Müritz weitgehend gemieden wurden. Diese Siedlungslage hatte möglicherweise wirtschaftliche Ursachen (Raseneisenerzgewinnung?). Am Beginn der späten Römischen Kaiserzeit ist ein weitgehender Siedlungsabbruch erkennbar, der wohl nur mit der Abwanderung größerer germanischer Bevölkerungsgruppen im Zuge der Völkerwanderung erklärt wer- 
den kann. Eine erneute starke Besiedlung des Müritzgebietes läßt sich erst für die slawische Periode belegen. Die Einwanderung slawischer Stämme erfolgte vermutlich im 7. Jh. n. Chr. Die zahlenmäßig stark angewachsenen jungslawischen Siedlungsplätze befinden sich im Gegensatz zu den altslawischen wieder in unmittelbarer Uferlage der Müritz. Flache Inseln und Halbinseln wurden als Schutzlagen oder Refugien genutzt. Für diese Zeit zeichnen sich bei Vipperow, Röbel und Waren drei Siedlungskonzentrationen ab, die als Siedlungskammern zu interpretieren sind. Erstere steht unzweifelhaft mit der für die 2. Hälfte des 12. Jh. überlieferten Terra Veprowe in Zusammenhang, deren Zentrum die Vipperower Burgwallinsel war (SCHOKNECHT 1993). Etwa um 1200 begann die starke Einwanderung deutscher Siedler, die mehrfach bereits bestehende slawische Ortschaften zur Ansiedlung nutzten, aber auch nahezu unbewohnte Areale z.B. am Ostufer urbar machten. Diese überwiegend erstmals im 13. Jh. erwähnten Dörfer und Städte prägen das heutige Siedlungsbild. Der niedere Adel erhielt die Erlaubnis, seine Rittersitze zu befestigen, wovon einige Turmhügel Zeugnis ablegen. Bereits im 14. Jh. erfaßte eine erste Wüstungsphase das Müritz-Umland.

Die für eine unterschiedliche Intensität der Uferbesiedlung u. a. herangezogenen Seespiegelschwankungen der Müritz (PREHN 1987) wurden inzwischen kritisch diskutiert, konnten jedoch als ursächlich nur teilweise bestätigt werden, beispielsweise für die jungslawische Periode (KAISER 1996b, 1998). Anzunehmen ist, daß durch den holozänen Wasserspiegelanstieg bis zum Subatlantikum (Slawenzeit) von ca. 4 $\mathrm{m}$ eine Reihe mesolithischer und neolithischer Seeuferstationen unter den Seespiegel geriet. Diese nur taucharchäologisch nachzuweisenden Fundplätze bergen zusammen mit den bekannten Feuchtbodenfundplätzen z.B. der Stinthorst oder der Vipperower Burgwallinsel ein großes siedlungsarchäologischlandschaftsgeschichtliches Potential!

\section{Zusammenschau und Ausblick}

In Abbildung 12 werden die Ergebnisse der vorliegenden Untersuchungen zueinander in Beziehung gesetzt. Nach dem gegenwärtigen Stand der geowissenschaftlichen und archäologischen Untersuchungen lassen sich erst wenige gesicherte Aussagen zum Verhältnis See und Mensch treffen, da einerseits die meisten nach der Seespiegelentwicklung zu erwartenden subaquatischen Fundplätze noch ihrer Entdeckung harren und andererseits bislang keine längeren Bohrkerne aus der Müritz verfügbar sind. Insbesondere an dem reliefstärke- ren und von Geschiebemergelstandorten geprägten Westufer mit seiner vergleichsweise höheren Siedlungsgunst dürften sich landseitig in Form von Kolluvien bzw. erodierten Flächen und seeseitig in Form eines Eintrages von Feinklastika bzw. anhand einer lokalen Eutrophierung deutliche Nutzungsspuren innerhalb verschiedener Siedlungsperioden nachweisen lassen.

Ein in seiner Datierung und hydrographischen Auswirkung gut abgesicherter Befund ist der wahrscheinlich auf Mühlenstau zurückzuführende Anstieg der Müritz von 62-63 m NN am Ende des 13. Jh. auf ca. $65 \mathrm{~m} \mathrm{NN}$ im 14. Jh. Die Überschwemmung großer Landflächen hat nicht nur zu einem zeitweiligen Verlust von Siedlungs- und Nutzungsflächen geführt, sondern muß auch gravierende Veränderungen im See selbst bewirkt haben. Im Großen Plöner See in Ostholstein, für den ein anthropogener Seespiegelanstieg von mehr als $2 \mathrm{~m}$ aus dem 13. Jh. bekannt ist, ließ sich eine an die Seeflächenvergrößerung und den dadurch erhöhten Stoffeintrag gekoppelte, später jedoch wieder abklingende Eutrophierung nachweisen (vgl. OHLE 1972, 1973). Ein ähnlicher Prozeß dürfte auch in der Müritz stattgefunden haben.

Der direkten und indirekten Wirkung des mittelalterlichen Menschen auf den See steht komplementär die Auswirkung landwirtschaftlicher Nutzung auf störungsempfindlichen Landstandorten am Ostufer gegenüber. Die Nutzungsintensivierung in der spätslawischen und insbesondere in der deutschmittelalterlichen bis neuzeitlichen Periode führte hier infolge von Winderosion zur Entstehung großflächiger „badlands“, die erst im 18./19. Jh. aufgeforstet wurden.

Bei Berücksichtigung der auf einer großen Fläche verteilten und mit unterschiedlicher Auflösung untersuchten Pollendiagramme sind die Beziehungen zwischen Mensch und Vegetation bisher nur näherungsweise zu fassen. Die Existenz alt- und mittelsteinzeitlicher Jäger- und Sammlergruppen läßt sich in den 3 bzw. 4 Pollendiagrammen nicht nachweisen. Die Verteilung der jüngeren Fundplätze im Untersuchungsgebiet und deren Korrelation mit den Pollendiagrammen muß fallweise betrachtet werden: Um Waren, Röbel und Rechlin liegende Fundplatzkonzentrationen sind wenigstens zum Teil auch „Bearbeiterareale“. Zum anderen wurde das Ostufer der Müritz zumindest in den vergangenen 100 Jahren überwiegend forstlich genutzt. In den dort auf trockenen Sandstandorten hauptsächlich stockenden Kiefernforsten war das Auffinden von archäologischen Fundplätzen nur unmittelbar nach dem Kahlschlag vor der Wiederaufforstung mög- 
lich. Im Bereich des Boeker Moores und des Profils Boek 1 sind bislang keine Fundplätze bekannt, so $\mathrm{da} ß$ sich die pollenanalytischen Aussagen zur Siedlungsgeschichte archäologisch nicht überprüfen lassen.

Die Interpretation des Pollendiagrammes Stinthorst, insbesondere des geringen Anteils von Fagus und Carpinus im Subatlantikum (IX-Xa), ist mit der relativ hohen Dichte eisen- und slawenzeitlicher Fundplätze gut vereinbar.

Am Prelitzsee wurde eine bronzezeitliche Siedlungsphase mit Rodungstätigkeit diagnostiziert. Die nächstgelegenen bekannten Fundplätze liegen ca. 4 km entfernt. Ausgedehnte Feldfluren erscheinen für diese Zeit uncharakteristisch. Es sind deshalb bisher noch nicht entdeckte Siedlungsplätze in der Nähe des Sees zu vermuten. Unmittelbar in der Nähe des Prelitzsees befindliche eisen- und kaiserzeitliche Fundplätze zeugen von den Verursachern der entsprechenden Siedlungszeiger-Werte im Pollendiagramm.

Für zukünftige landschaftsgeschichtliche Untersuchungen unterschiedlicher Disziplinen im Müritzgebiet lassen sich u.a. die folgenden Forschungsschwerpunkte bzw. Desiderata formulieren:

- Geologisch-geomorphologische Entwicklung im Pleistozän, insbesondere im Weichselhoch- und Spätglazial.

- Auffindung und Datierung der aus verschiedenen Quellen bekannten subaquatischen Terrassen der Müritz.

- Paläoökologische Bearbeitung von Bohrkernen aus der Müritz mit guter Auflösung des Holozäns. In diesem Zusammenhang Erstellung eines Standardpollendiagramms für die Region.

- Aussagen zur weichselspätglazialen Vegetationsentwicklung u.a. im Hinblick auf geomorphologische, insbesondere äolische Prozesse.

- Nachweis und Folgen ur- und frühgeschichtlicher sowie historischer Landnutzung am siedlungsgünstigen Westufer.

- Archäologische und palynologische Untersuchung von Feuchtbodensiedlungen sowie archäologische Unterwasserprospektion.

- Kalibrierte ${ }^{14} \mathrm{C}$-Chronologie aller Ereignisse.

- Systematische Erfassung der Ausprägung und Folgen neuzeitlicher und moderner Landnutzung auf die Müritz und ihre Ufer.

\section{Schriftenverzeichnis}

Andersen, S. T. (1986): Palaeoecological studies of terrestrial soils. - In: BERGLUND, B. E. [Ed.]: Handbook of Holocene palaeoecology and palaeohydrology: 165-177; Chichester.

Andersen, S. T. (1998): Pollen analytical investigations of barrows from the Funnel Beaker and Single Grave Cultures in the Vroue area, West Jutland, Denmark. - Journal of Danish Archaeology 1994/ 95, 12: 107-132.

AndREs, W. (1998): Terrestrische Sedimente als Zeugen natürlicher und anthropogener Umweltveränderungen seit der letzten Eiszeit. - Verhandlungen zum 51. Deutschen Geographentag, Bonn 1997: 118-133; Stuttgart.

Berglund, B. E. [Ed.] (1991): The cultural landscape during 6000 years in southern Sweden - the Ystad Project. - Ecological Bulletins, 41; Kopenhagen.

Billwitz, K., Helbig, H., Kaiser, K., De Klerk, P., KÜHN, P. \& Terberger, T. (2000): Untersuchungen zur spätpleistozänen bis frühholozänen Landschafts- und Besiedlungsgeschichte in Mecklenburg-Vorpommern. - Neubrandenburger Geologische Beiträge, 1: 24-38.

BleILE, R. (2000): Unterwasserarchäologische Voruntersuchungen an der Burgwallinsel Vipperow in der Müritz mit dem Fund eines Einbaumes. - Archäologische Berichte aus Mecklenburg-Vorpommern, 7: $151-157$.

Brande, A. (1995): Moorgeschichtliche Untersuchungen im Spandauer Forst (Berlin). - Schriftenreihe für Vegetationskunde, 27 (Festschrift Sukopp): 249255.

Deppe, H.-J. \& Prill, H. (1958): Ein Beitrag zur Geschichte der Müritz. - Archiv der Freunde der $\mathrm{Na}$ turgeschichte in Mecklenburg, 4: 116-148.

Dieckmann, O. \& Kaiser, K. (1998): Pedologische und geomorphologische Befunde zur historischen $\mathrm{Bo}-$ denerosion im Müritz-Nationalpark, MecklenburgVorpommern. - In: Asmus, I., PoradA, H. T., \& SCHLEINERT, D. [Hrsg.]: Geographische und historische Beiträge zur Landeskunde Pommerns. - 5965; Schwerin.

Duphorn, K., Kliewe, H., Niedermeyer, R.-O., JanKE, W., \& Werner, F. (1995): Die deutsche Ostseeküste. - Sammlung geologischer Führer, 88; Berlin u. Stuttgart.

Engmann, K. F. (1937): Pollenanalytische Untersuchungen fossiler Böden im Flugsandgebiet von Leussow (Südwestmecklenburg). - Mitteilungen der Mecklenburgischen Geologischen Landesanstalt N. F., 10: 1-24.

FIrBAS, F. (1949): Die spät- und nacheiszeitliche Waldgeschichte Mitteleuropas nördlich der Alpen. Bd. 1 Allgemeine Waldgeschichte. - Jena.

Frenzel, B. (1996): Bericht Frenzel der Projektgruppe „Terrestrische Paläoklimatologie“ im Rahmen des Forschungsschwerpunktes „Klimavariabilität und Erkennung des menschlichen Signals" des Bundesministeriums für Bildung, Wissenschaft, Forschung und Technologie. - Jahrbuch der Aka- 
demie der Wissenschaften und der Literatur Mainz, 1995: 244-305.

Geinitz, E. (1886): Die Seen, Moore und Flußläufe Mecklenburgs. - Güstrow.

Geinitz, E. (1913): Die großen Schwankungen der norddeutschen Seen. - Die Naturwissenschaften, 1: 665-670.

Geyh, M. (1983): Physikalische und chemische Datierungsmethoden in der Quartär-Forschung. Clausthaler Tektonische Hefte, 19: $163 \mathrm{~S}$.

Gringmuth-Dallmer, E. (1997): Das Projekt „Mensch und Umwelt im Odergebiet in ur- und frühgeschichtlicher Zeit". Eine Zwischenbilanz. - Bericht der Römisch-Germanischen Kommision, 78: 5-27.

Harrison, S. P., Yu, G. \& Tarasov, P. E. (1996): Late Quaternary lake-level record from northern Eurasia. - Quaternary Research, 45: 138-159.

Hollnagel, A. \& SchoKnecht, U. (1956): Die Burgwallinsel bei Vipperow, Kreis Röbel. - Bodendenkmalpflege in Mecklenburg, Jahrbuch 1954: 121-142.

JAGER, K.-D. (1987): Zum gegenwärtigen Kenntnisstand über nacheiszeitliche Wasserstandsschwankungen an Binnenseen im Jungmoränengebiet des nördlichen Mitteleuropa. - Wissenschaftliche Zeitschrift der Ernst-Moritz-Arndt-Universität Greifswald, math.-nat. R., 36: 44-48.

JESCHKE, L. (1997): Seen und Moore in MecklenburgVorpommern aus landschaftsökologischer Sicht. Greifswalder Geographische Arbeiten, 14: 33-50.

KAISER, K. (1996a): Paläohydrologische und geomorphologische Untersuchungen zur holozänen Landschaftsentwicklung im Müritz-Nationalpark, Mecklenburg-Vorpommern. - Unveröff. Dipl.-Arb., Universität Greifswald, Geographisches Institut.

KAISER, K. (1996b): Zur hydrologischen Entwicklung mecklenburgischer Seen im jüngeren Quartär. Peterm. Geogr. Mitt., 140: 313-332.

KAISER, K. (1998): Die hydrologische Entwicklung der Müritz im jüngeren Quartär - Befunde und ihre Interpretation. - Zeitschrift für Geomorphologie, N.F., Suppl.-Bd., 112: 143-176.

Kaiser, K., Terberger, T. \& Jantzen, C. (2000): Rivers, lakes and ancient men: Relationships of palaeohydrology and the archaeological record in Mecklenburg-Vorpommern (North-East-Germany). Beiträge zur Ur- und Frühgeschichte MecklenburgVorpommerns, 35: 405-409.

KLoss, K. (1980): Pollenanalysen zur Vegetations-, Siedlungs- und Moorgeschichte am Südrand der ostmecklenburgisch-brandenburgischen Seenplatte (Kreis Gransee). - Archiv für Naturschutz und Landschaftsforschung, 20: 203-212.

Knapp, H. D., Voigtländer, U. \& Grundmann, L. (1999): Das Müritzgebiet. Ergebnisse der landeskundlichen Bestandsaufnahme im Raum Waren, Klink, Federow und Rechlin. - 282 S.; Weimar.

Kossack, G., Behre, K.-E. \& Schmid, P. (1984): Archäologische und naturwissenschaftliche Untersuchungen an ländlichen und frühstädtischen Siedlungen im deutschen Küstengebiet vom 5. Jahrhundert v. Chr. bis zum 11. Jahrhundert n. Chr., Bd. 1: Ländliche Siedlungen. - 461 S.; Weinheim.
Kretschmer, H., Arndt, K. \& Müller, H. M. (1971): Untersuchungen an Dünen im Gebiet des Dänengrundes bei Zempin (Usedom). - Peterm. Geogr. Mitt., 115: 9-15.

Lange, E., JeschKe, L. \& Knapp, H. D. (1986): Die Landschaftsgeschichte der Insel Rügen seit dem Spätglazial. Ralswiek und Rügen - Landschaftsentwicklung und Siedlungsgeschichte der Ostseeinsel, Teil I. - 174 S.; Berlin.

LFG M-V (in Vorb.): Erläuterungsband zur forstlichen Standortskarte für den Müritz-Nationalpark. Landesamt für Forsten und Großschutzgebiete Mecklenburg-Vorpommern. - Malchin.

Mangerud, J., Andersen, S. T., Berglund, B. \& DonNER, J. J. (1974): Quaternary stratigraphy of Norden, a proposal for terminology and classification. - Boreas, 3: 109-128.

Marcinek, J., Seifert, S. \& Zaumseil, L. (1996): Zur Bildung und Entwicklung des Gewässernetzes in Brandenburg. - Gewässerökologie Norddeutschlands, 2: 7-21.

Martens, W. (1955): Norddeutschlands Seen - Ein Ergebnis der Eiszeit. - Jena.

Merkt, J. \& Müller, H. M. (1999): Varve chronology and palynology of the Lateglacial in Northwest Germany from lacustrine sediments of Hämelsee in Lower Saxony. - Quaternary International, 61: 41-59.

Müller, A. (1999): Die Müritz. Die Geschichte einer Landschaft und ihrer Bewohner rund um Deutschlands größten Binnensee. - 236 S.; Waren/Müritz.

Mülder, H. M. \& KoHL, G. (1966): Radiocarbondatierungen zur jüngeren Vegetationsentwicklung Südostmecklenburgs. - Flora, 156: 408418.

Müller, H. M., Kopp, D. \& KoHL, G. (1971): Pollenanalytische Untersuchungen zur Altersbestimmung von Humusauflagen einiger Bodenprofile im subkontinentalen Tieflandgebiet der DDR. - Peterm. Geogr. Mitt., 115: 25-36.

Müller-Wille, M., Higelke, B., Hoffmann, D., Menke, B., Brande, A., Bokelmann, K., Saggaus, H. E. \& KüHN, H. J. (1988): Norderhever-Projekt. 1 Landschaftsentwicklung und Siedlungsgeschichte im Einzugsgebiet der Norderhever (Nordfriesland). - 232 S.; Neumünster.

Niewiarowski, W., Noryskiewicz, B., Piotrowski, W., \& SinKIEWICZ, M. (1995): An outline of natural and anthropogenic changes of geographical environment in the Biskupin area during the last 7000 years. - Quaternary Studies in Poland, 13: 77-88.

Ohle, W. (1972): Die Sedimente des Großen Plöner Sees als Dokumente der Zivilisation. - Jahrbuch für Heimatkunde Plön, 2: 7-27.

OHLE, W. (1973): Die rasante Eutrophierung des GroBen Plöner Sees in frühgeschichtlicher Zeit. - Die Naturwissenschaften, 60: $47 \mathrm{~S}$.

Prehn, B. (1985): Die ur- und frühgeschichtliche Fundplatzverteilung im Umland der Müritz sowie ihre besiedlungs- und landschaftsgeschichtliche Aussage. - Unveröff. Dipl.-Arb., Humboldt-Universität Berlin, Institut für Ur- und Frühgeschichte. 
Prehn, B. (1987): Zu Hinweisen auf Seespiegelschwankungen der Müritz. - Wissenschaftliche Zeitschrift der Ernst-Moritz-Arndt-Universität Greifswald, math.-nat. R., 36: 49-51.

Ruchнӧғт, F. (1999): Der Wasserstand der „Oberen Seen" in Mecklenburg in Mittelalter und früher Neuzeit. - Archäologische Berichte aus Mecklenburg-Vorpommern, 6: 195-208.

Rust, A. (1937): Das altsteinzeitliche Rentierlager Meiendorf. - 146 S; Neumünster.

Schlichterle, H. (1990): Aspekte der siedlungsarchäologischen Erforschung von Neolithikum und Bronzezeit im südwestdeutschen Alpenvorland. Berichte der Römisch-Germanischen Kommission, 71: 208-244.

SCHOKNECHT, U. (1959): Der mittelsteinzeitliche Wohnplatz „Stinthorst" bei Waren. - Bodendenkmalpflege in Mecklenburg, Jahrbuch 1957: 7-24.

SCHOKNECHT, U. (1993): Neue Funde und Befunde von der jungslawischen Burgwallinsel Vipperow, Kreis Röbel. - Bodendenkmalpflege in MecklenburgVorpommern, Jahrbuch 1992: 213-260.

Schoknecht, T. (1996): Pollenanalytische Untersuchungen zur Vegetations-, Siedlungs- und Landschaftsgeschichte in Mittelmecklenburg. - Beiträge zur Ur- und Frühgeschichte Mecklenburg-Vorpommerns, 29: 68 S.; Lübstorf.

SchumaCHeR, C. W. C. (1790): Über den sogenannten Röbelschen Wald unter dem Wasser in der Müritz. - Monatschrift von und für Mecklenburg, 3: 246251.

Wiethold, J. (1998): Studien zur jüngeren postglazialen Vegetations- und Siedlungsgeschichte im östlichen Schleswig-Holstein. - Universitätsforschungen zur prähistorischen Archäologie, 45: 365 S.; Bonn.

Yu, G., \& Harrison, S. P. (1995): Holocene changes in atmospheric circulation patterns as shown by lake status changes in northern Europe. - Boreas, 24: 260-268. 